\title{
11. Thomas Reid
}

\author{
Johannes Haag und Markus Wild
}

\subsection{Einführung: Reids Begriff des Common Sense}

Wer Berkeleys These vernimmt, dass es keine materiellen Dinge gibt, oder Descartes zuhört, der sich seiner Existenz versichert, reagiert vielleicht mit Kopfschütteln und Lachen. Dies, so würde Reid sagen, ist die natürliche und richtige Reaktion des Common Sense auf eine falsche Philosophie. In Reids Augen muss die Ideentheorie, da sie zwangsläufig in den Skeptizismus führt, dem Common Sense widerstreiten. Normale, reife Menschen glauben ganz natürlich, dass sie materielle Dinge sehen und dass sie selbst existieren, dafür brauchen sie keine Beweise. Philosophen sollten dem Skeptiker auch keine Beweise dafür geben. Die Überzeugung, dass vor mir ein Baum steht, ist für sich allein gewisser, als alle Thesen des Ideentheoretikers zusammen, die zur Relativierung oder gar zur Negation dieser Gewissheit führen. Aber weshalb sollte sich ein Skeptiker mit meiner Behauptung zufrieden geben, dass ich gewiss einen Baum vor mir stehen sehe? Wie lautet mein Schluss auf die Existenz des Baums? Doch solche Fragen des Skeptikers sollen gerade nicht beantwortet werden. Reid ist der Ansicht, dass wir nicht durch Vernunftschlüsse dazu gebracht werden, an die Existenz wahrgenommener Dinge zu glauben, sondern durch unsere natürlichen Wahrnehmungsvermögen. ${ }^{1}$

Aber gebietet uns nicht die Vernunft, dass wir uns des Urteils über die Existenz des Baums enthalten, wenn wir seine Existenz nicht beweisen können? Reid zufolge wird die Vernunft gegenüber anderen Vermögen einseitig privilegiert: ${ }^{2}$ „Wenn unsere Vermögen uns täuschen, warum

1 Vgl. Essays 2.5, 258; 2.21, 332. Reids Überlegung gleicht G. E. Moores Beweis für die Außenwelt. Moore meint, dass seine Überzeugung, dass er zwei Hände hat, gewisser ist, als die Prämissen des Skeptikers, der dies bezweifelt, vgl. Moore 1993; Greco 2003.

2 Vgl. die Deduktionsthese in der Einleitung zu Bd. 1, 11.1.2. 
sollten sie uns bei einem Schluss nicht ebenso täuschen wie bei einer anderen Tätigkeit?“ (Essays 4.4, 447) Normalerweise vertrauen wir allen kognitiven Vermögen gleichermaßen. Deshalb optiert Reid gegen die Ideentheorie und für den Common Sense. (Seine Philosophie wird deshalb als Philosophie des Common Sense verstanden.) In der Betrachtung der Reidschen Auffassung des Common Sense werden wir feststellen, dass sie zweideutig ist. Der Grund für diese Zweideutigkeit besteht darin, dass sich Reid nicht ganz von jenem falschen Philosophieverständnis frei gemacht hat, das er der Ideentheorie vorhält.

Die Philosophie des Common Sense ist Reids methodologischer Gegenentwurf zur Ideentheorie, die zuerst als Irrweg erkannt werden muss. Warum aber muss das Ideensystem als Irrweg verstanden worden sein, um eine Philosophie des Common Sense entwickeln zu können? Nun, Reid glaubt, dass die Ideentheoretiker nicht nur falschen Thesen, sondern auch einer falschen Auffassung von Philosophie anhängen. Erstens achten Ideentheoretiker zu wenig auf die Sprache. Nicht nur verwenden sie Begriffe (wie etwa den Begriff der Idee) vieldeutig oder abweichend vom üblichen Gebrauch, sie gehen auch der Oberflächengrammatik der Sprache auf den Leim. So legt es etwa die Struktur der Sprache nahe, dass wir uns mit geistigen Zuständen immer auf Objekte beziehen (vgl. systematischen Essay, 11.3). Zweitens wählen Ideentheoretiker den Weg der Analogie und nicht den Weg der Reflexion (Inquiry 7, 201; Bd. 1, S. 414 f.). Sie analogisieren den Geist mit Körpern und glauben, dass dem Geist äußere Gegenstände nur über Vermittler präsent sein können, wie zwischen Körpern nur über Vermittler kausale Interaktionen stattfinden können. Die richtige Philosophie betrachtet geistige Phänomene als Phänomene sui generis und widmet sich ihnen über den Weg der Reflexion. Drittens schließlich zielen Ideentheoretiker auf den Bau eines Systems, ohne auf eine vorherige sorgfältige Analyse einzelner Phänomene zu achten (Inquiry 1.2, 99; Bd. 1, S. 403). Deshalb neigen sie dazu, Resultate aus einem bestimmten Phänomenbereich auf andere zu übertragen und dadurch zu verfälschen. Erst wenn wir die sprachlichen, analogischen und systematischen Verzerrungen erkennen, die die Ideentheorie unserem Denken aufgeprägt hat, können wir zur wahren Philosophie gelangen. Diese Philosophie besteht nicht in der Akklamation der Meinungen der Menge. Sogar wenn Reid die Vertreter der Ideentheorie ostentativ zum Gespött der Menge macht, hat dies eine philosophische Funktion, denn Theorien, die dem Common Sense widersprechen, „kann man von Irrtümern durch folgendes Mittel unterscheiden: sie sind nicht nur falsch, sondern absurd. Die Natur hat uns eine bestimmte 
emotionale Reaktion gegeben, mit der wir die Absurdität zurückweisen, nämlich die Lächerlichkeit" (Essays 4.4, 438). Die Meinungen der Ideentheoretiker sind lächerlich, weil sie dem Common Sense widerstreiten. „Common Sense“ ist jedoch ein vieldeutiger Begriff. Personen mit „gesundem Menschenverstand“ haben eine unüberblickbare Menge von Meinungen, wie etwa, dass es besser ist, bei Gewittern nicht über Gebirgskämme zu gehen, oder dass man nicht am weiter entfernten Rand eines Glases trinkt. Man schreibt jemandem auch gesunden Menschenverstand zu, wenn er Situationen und Personen richtig zu beurteilen vermag. Spezifischer jedoch meint „Common Sense“ eine Menge nicht begründbarer Grundsätze (principles), die sich in allen reifen, normalen Menschen finden, und in diesem spezifischen Sinn verwendet Reid den Begriff. Primär bestimmt Reid den Common Sense als ein Urteilsvermögen, nämlich als ,jenes Maß an Urteilsvermögen, das den Menschen gemein (common) ist, mit denen wir uns unterhalten und mit denen wir uns auf Unternehmungen einlassen“ (Essays 6.2, 421). Common Sense ist ein Vermögen zur Beurteilung von ersten Grundsätzen als erste Grundsätæe. Der Common Sense entdeckt, ,was wahr und was falsch ist bezüglich selbstevidenter Dinge“, denn ,alle Erkenntnis und alle Wissenschaft muss auf selbstevidenten Grundsätzen beruhen" (Essays 6.2, 422). In diesem Sinne versteht Reid unter "Common Sense“ auch eine Menge von ersten Grundsätzen.

Obschon Reid behauptet, dass erste Grundsätze allen Menschen gemeinsam sind, leugnet er nicht, dass wir uns darüber streiten können, welche Grundsätze dazugehören und welche nicht, und dass wir erste Grundsätze bestreiten können. ${ }^{3}$ Wichtig ist für Reid die Beobachtung, dass man am Ende der argumentativen Möglichkeiten angekommen ist, wenn man sich über erste Grundsätze uneins wird. Man ruft aus: „Aber das ist doch selbstverständlich!" und bricht die Debatte ab. Abbruch der Debatte bedeutet aber nicht Abbruch des Gesprächs, denn es gibt durchaus Mittel und Wege, sich über erste Grundsätze zu verständigen. Von den ersten Grundsätzen und dem Common Sense als Urteilsvermögen muss man nämlich diejenigen Strategien unterscheiden, die Reid aufzählt, um den Common Sense gegen seine Verächter ins Recht zu setzen. Wir haben das Mittel der Lächerlichkeit bereits erwähnt. Reid verweist

\footnotetext{
3 Zwar hat der Ausdruck „Common Sense“ Reid zufolge eine klare Bedeutung (so wie man weiß, was man mit „Bayern“ bezeichnet), aber es ist unklar, was alles zum Common Sense gehört. Gehört Lindau am Bodensee zu Bayern? Reids Beispiel ist York County (Essays 6.2, 423).
} 
weiter auf ad-hominem-Argumente („Warum akzeptierst Du diese, aber nicht jene Grundsätze?"), auf die Möglichkeit einer Reductio ad absurdum (,Wenn Du das bestreitest, kannst Du nicht mehr glauben, dass p, und das ist absurd!“), auf den Konsens der Gebildeten und Ungebildeten aller Zeiten, auf den allgemeinen Sprachgebrauch, auf Grundsätze, die wir als angeboren betrachten müssen, und auf die praktische Unverzichtbarkeit gewisser Grundsätze. Selbstevidente Grundsätze können nicht direkt bewiesen oder begründet, sondern nur indirekt aufgezeigt werden.

Ist der Common Sense nun nicht eher Bestandteil des Vernunftvermögens? In gewisser Weise ja, denn Reid unterscheidet zwei Grade der Vernunft:

Wir schreiben der Vernunft zwei Aufgaben oder Grade [degrees] zu. Die erste besteht darin, über selbstevidente Dinge zu urteilen [to judge of things self-evident], und die zweite darin, daraus Schlüsse zu ziehen. Das erste ist der Bereich des Common Sense, und zwar sein alleiniger Bereich ... [Common Sense] ist nur ein anderer Name für einen Zweig oder einen Grad der Vernunft. (Essays 6.2, 425)

Reid bestimmt Vernunft als das Vermögen Schlüsse zu ziehen. Selbstevidente Prinzipien werden aber nicht aus anderen abgeleitet, da sie ja selbstevident sind. Der Common Sense darf also nicht als Vermögen des Schließens betrachtet werden, er ist auch nicht das Vermögen, aus ersten Grundsätzen Schlüsse zu ziehen. ${ }^{4}$ Bei gesunden Personen entwickelt sich der Common Sense wie das Sehvermögen oder das Gehen. Die Vernunft hingegen wird durch Unterweisungen und Regeln eingeübt, aber nur, wenn der Common Sense vorhanden ist. Der Common Sense kann deshalb als „Grad der Vernunft“ bezeichnet werden, weil nur er uns zu rationalen Wesen macht. Der Common Sense als Vermögen die Wahrheit und Falschheit selbstevidenter Grundsätze zu beurteilen, ist nämlich grundlegender als das Vermögen zu schließen. Selbstevident bedeutet einfach „nicht abgeleitet“. Der Common Sense urteilt, dass Grundsätze selbstevident sind, er urteilt nicht mithilfe selbstevidenter Dinge. Auch ist der Common Sense nicht das Vermögen, erste Grundsätze hervorzubringen, denn er kann selbstevidente und nicht abgeleitete Grundsätze nicht erzeugen, sondern nur aufspüren und als solche beurteilen. ${ }^{5}$ Schließlich ist der Common Sense kein Vermögen zur Aufdeckung notwendiger oder analytischer Grundsätze, denn Reid betrachtet auch kontingente Wahrheiten als erste Grundsätze (Essays 6.5). Der

\footnotetext{
${ }^{4}$ Gegen die These von Lehrer 1989, 152.

5 Gegen die Deutung von Rysiew 2003, 29-30.
} 
Common Sense ist also ein Vermögen zur Beurteilung von ersten (notwendigen oder kontingenten) Grundsätzen als selbstevident und nicht abgeleitet, d. h. er beurteilt erste Grundsätze als erste Grundsätze.

Wie steht es mit dem Common Sense als Menge von Grundsätzen? Reid nennt zwölf kontingente Grundsätze des Common Sense (Essays 6.5, 442-452): (1) Alles, dessen ich mir bewusst bin, existiert. (2) Die Gedanken, deren ich mir bewusst bin, sind meine Gedanken. (3) Jene Dinge, an die ich mich deutlich erinnere, sind wirklich geschehen. (4) Unsere Identität als Person reicht so weit zurück wie wir uns deutlich erinnern. (5) Jene Dinge, die wir mit unseren Sinnen deutlich wahrnehmen, existieren wirklich, und sie existieren so, wie wir sie wahrnehmen. (6) Wir haben Macht über unsere Tätigkeiten und unseren Willen. (7) Die natürlichen Vermögen, durch die wir Wahres von Falschem unterscheiden, sind nicht trügerisch. (8) In unseren Mitmenschen ist Leben und Verstand. (9) Das Verhalten der Mitmenschen zeigt ihre Gedanken und Neigungen an. (10) Das Zeugnis anderer über Tatsachen wird mit Achtung aufgenommen. (11) Das Verhalten von Personen folgt gewissen wahrscheinlichen Regelmäßigkeiten. (12) In der Natur ist dasjenige, was war, unter gleichen Umständen wahrscheinlich demjenigen ähnlich, was sein wird. Einige Sätze sind nach Reids Auffassung von Ideentheoretikern als erste Grundsätze ausgewiesen worden, beispielsweise (1) von Descartes oder (12) von Hume. Einige Sätze müssen mit dem richtigen $\mathrm{Ohr}$ gehört werden. So meint Reid mit (3) nicht, dass ein subjektives Gefühl einer deutlichen Erinnerung die Existenz der erinnerten Ereignisse garantiere, sondern vielmehr, dass es begrifflich so ist, dass erinnerte Ereignisse ipso facto wirkliche Ereignisse sind, andernfalls handelt es sich nicht um Erinnerungen.

Betrachten wir (7) genauer. Warum sollte ich meinem Gedächtnis, den Sinnen, dem Bewusstsein, dem Zeugnis anderer usw. überhaupt Vertrauen schenken? Reid meint, dass die natürlichen Vermögen, durch die wir Wahres von Falschem unterscheiden, nicht trügerisch sind. Bei (7) scheint es sich um einen Meta-Grundsatz zu handeln, der nicht auf derselben Ebene angesiedelt ist wie die anderen. Reid versucht die Unabdingbarkeit des Vertrauens in alle unsere kognitiven Vermögen auf verschiedenen Wegen zu begründen. Hier ist einer dieser Wege: Reid unterscheidet zwei Arten von Skeptikern (Inquiry 5.7, 130; Bd. 1, S. 412-414). Der Totalskeptiker zweifelt an allen Überzeugungen, solange nicht die Zuverlässigkeit aller kognitiver Vermögen bewiesen ist. Ihm wirft Reid nicht nur vor, dass es psychisch und praktisch unmöglich ist, keine Überzeugungen zu haben. Da dieser Skeptiker gar keine Über- 
zeugungen hat, besitzt er natürlich keine Ausgangsbasis für einen Beweis der Zuverlässigkeit seiner Vermögen. Deshalb kann er weder Gründe für seine Zweifel noch gegen meine Überzeugungen angeben, akzeptiert er doch das Vermögen, Gründe zu geben, nicht als zuverlässig (Inquiry 5.7, 130; Bd. 1, S. 413; Essays 6.5, 447). Eine totale Skepsis ist also nicht vertretbar. Der Semiskeptiker hingegen akzeptiert einige Überzeugungen und betrachtet sie als Ausgangspunkt für die Beurteilung der Zuverlässigkeit seiner anderen kognitiven Vermögen. Er akzeptiert beispielsweise die Vertrauenswürdigkeit seines Vernunftvermögens und die Überzeugung, dass er existiert und denkt, alles andere muss abgeleitet werden. Reid wirft dem Semiskeptiker vor, dass er seine Überzeugungen arbiträr wählt und sein Vertrauen willkürlich verschenkt (Inquiry 6.20, 183). Deshalb gibt es, seiner Auffassung nach, keine guten Gründe, dem Vorgehen des Semiskeptikers zu folgen. Es bleibt nur noch eine Option: Wir müssen allen unseren Vermögen gleichermaßen vertrauen. ${ }^{6}$ Das bedeutet nicht, dass die Vermögen nie etwas hervorbringen, das falsch oder irreführend ist. Aber wenn wir dies überhaupt feststellen wollen, müssen wir die Zuverlässigkeit von anderen kognitiven Vermögen grundsätzlich akzeptieren. In diesem Sinne ist (7) ein Meta-Grundsatz.

Nun stellt sich eine wichtige Frage: Sind diese Grundsätze explizit geglaubte Grundsätze, die wir mit allen reifen und normalen Menschen teilen, oder handelt es sich um impliz̨ite Grundsätze, die wir für all unser praktisches und theoretisches Tun stillschweigend akzeptieren müssen?7 Vieles von dem, was Reid sagt, deutet darauf hin, dass er sie für explizite, geteilte Überzeugungen hält, auf denen wir unsere weiteren Überlegungen aufbauen. Reid teilt also ein fundamentalistisches Bild der Erkenntnis. Seine Begründung für die Existenz erster Grundsätze lautet ja, dass Erkenntnis auf nicht abgeleiteten Grundsätzen beruhen müsse. Anders als Descartes, der das Fundament zu schmal anlegt und nur Grundsatz (1) vertraut, möchte Reid mit (7) ein breites Fundament. Aber müssen wir tatsächlich alle unsere Erkenntnisse aus expliziten Grundsätzen ableiten, unsere Praktiken auf expliziten Prinzipien gründen? An vielen Stellen sagt Reid, dass erste Grundsätze „,bei den meisten Menschen ihre Wirkungen hervorbringen, ohne dass man je auf sie achtet oder zum Gegenstand des Nachdenkens macht. Kein Mensch denkt je an diesen Grundsatz

${ }^{6}$ Vgl. DeRose 1989, $326 \mathrm{ff}$.

7 Es stellt sich ein zweite wesentliche Frage, die hier aber nicht behandelt werden soll: Können wir einfach nicht umhin, diese Grundsätze für wahr zu halten, oder sind sie auch tatsächlich wahr? Für einen Versuch vgl. de Bary 2002, Kap. 9. 
[(7)], solange er nicht über den Ursprung des Skeptizismus nachdenkt, und dennoch beherrscht es immerzu seine Meinungen." (Essays 6.5, 448) Wir sind genötigt, die Grundsätze ,im Alltag stillschweigend zu akzeptieren [must take for granted], ohne dass wir dafür Gründe angeben können" (Inquiry 2.6, 108). Aber etwas stillschweigend zu akzeptieren, das unser Denken und Tun immerzu beherrscht, scheint nicht dasselbe zu sein, wie bestimmte Sätze zu glauben, und es scheint nicht dasselbe zu sein wie ein Fundament nicht abgeleiteter Prinzipien, von denen wir durch Schlüsse Erkenntnisse und die Regeln unseres Tun ableiten. Einerseits versteht Reid die Grundsätze des Common Sense als explizite, geteilte Sätze, die wir tatsächlich glauben und auf denen wir aufbauen, andererseits fasst er sie als etwas auf, das wir in unserem Denken und Tun stets stillschweigend akzeptieren. Im ersten Verständnis, das Reids offizieller Position entspricht, sind Grundsätze eine Art Untergrund. Im zweiten Verständnis, das sich wie ein inoffizieller roter Faden durch Reids Ausführungen zeiht, sind sie eine Art Hintergrund. ${ }^{8}$

Das offizielle Bild lebt von einer Analogie zwischen unserem Wissen und dem Errichten eines Gebäudes. Reid scheint anzunehmen, dass wir die ersten Grundsätze gleichsam vor dem Geist haben müssen, um ihnen folgen zu können. Schließlich suggeriert das Bild vom Wissensgebäude, dass wir unser Wissen aus ersten Prinzipien deduzieren können. Eine Analogie leitet also Reids Explikation der ersten Grundsätze. Er scheint mit den Ideentheoretikern der Ansicht zu sein, dass diese Grundsätze wie Ideen dem Geist gegenwärtig sein müssen, und dass wir aus ihnen alles Weitere ableiten können. Im Bild, dem Reid offiziell folgt, sind erste Grundsätze so etwas wie Anleitungen oder regulative Prinzipien für unser Denken und Tun, die unabhängig von diesem Denken und Tun sind. Genau dies ist aber die Sichtweise des Skeptikers, der Reid doch entgegentreten möchte. Denn der Skeptiker zieht die Grundsätze des Common Sense in Zweifel und betrachtet sie dabei als etwas, das von seiner Tätigkeit des Zweifelns unabhängig ist. Es sieht mithin so aus, als würde Reid hier in jene falsche Philosophie verfallen, die er den Ideentheoretikern vorgeworfen hat.

Was besagt das inoffizielle Bild? Der Common Sense beurteilt selbstevidente Grundsätze nicht als selbstevident, um sie danach in seinem

${ }^{8}$ Diese Überlegungen halten sich an die Analyse von Wolterstorff 2001, Kap. 9. Wolterstorff ist darüber hinaus auch noch der Ansicht, dass Reid sich anschickt, mit dem roten Faden etwas auszuarbeiten, das wir erst durch Wittgenstein zu verstehen gelernt haben; vgl. ebd. 231-244. 
Denken und Tun anzuwenden. Vielmehr sind diese Grundsätze konstitutiv für unser Denken und Tun, und deshalb sind unser Denken und Tun und die Grundsätze nicht voneinander unabhängig. Wir denken und handeln, indem wir die Grundsätze des Common Sense stillschweigend akzeptieren. Man kann sich den Unterschied zwischen den beiden Bildern anhand einer Unterscheidung von regulierenden Regeln und konstitutiven Regeln vor Augen führen. Die Regeln, wie man im öffentlichen Raum mit heftigen Emotionen umgeht oder wem man auf der Straße den Vortritt lassen soll, sind bloß regulierend, denn sie regeln die unabhängig von ihnen existierende Emotionen bzw. den Verkehr. Das Schachspiel hingegen existiert nicht unabhängig von den Regeln des Schachspiels, Schachspielen heißt diesen Regeln zu folgen. Wer den König wie eine Dame führt, der spielt nicht schlecht oder ungewöhnlich Schach, sondern gar nicht. Die Grundsätze des Common Sense gleichen solchen konstitutiven Regeln. Im Unterschied zu den konstitutiven Regeln des Schachspiels beruhen die Grundsätze des Common Sense jedoch nicht auf einer definitiven Menge expliziter Regeln. Vor allem aber konstituieren die Grundsätze nicht nur einen bestimmten Bereich unseres Denkens und Tuns, sondern sie bestimmen unser Denken und Tun überbaupt. ${ }^{9}$ Reid vergleicht die ersten Grundsätze des Common Sense bisweilen mit wissenschaftlichen Axiomen (Essays 1.2, 230, Inquiry 5.7, 130). In diesem konstitutiven Sinne sind die Grundsätze des Common Sense quasi-axiomatische Konstanten unseres Denkens und Tuns, die normale, reife Personen stillschweigend als Hintergrund akzeptieren.

\subsection{Stellenkommentar}

\subsubsection{Auszüge aus An Inquiry into the Human Mind on the Principles of Common Sense / Eine Untersuchung über den menschlichen Geist nach den Prinzipien des Common Sense (1769) (zitiert als: Inquiry)}

K1: Descartes' Wirbeltheorie versucht die Bewegungen der Himmelskörper zu erklären. Ein Wirbel (vortex) ist ein kreisender Strom materieller Teilchen. Die Planeten und Kometen bewegen sich auf solchen Strömen (vgl. Principia philosophiae III). Descartes' Theorie sollte mit der

9 Zur Unterscheidung zwischen regulierenden und konstitutiven Regeln vgl. Searle 2004, Kap. 6. Für eine Anwendung auf Reid vgl. Rysiew 2003. Searle 2004, Kap. 4 verwendet auch den Begriff des Hintergrunds. 
mechanistischen Naturphilosophie kompatibel sein. Sie galt in der ersten Hälfte des 18. Jh. als Konkurrentin von Newtons Theorie der Gravitation, vermochte aber deren mathematische Präzision und empirische Testbarkeit nicht zu erreichen (vgl. auch Essays 4.2; Bd. 1, S. 433). Der Ausdruck ,archeus“ wird von Vertretern der frühneuzeitlichen Iatrochemie (wie Paracelsus oder Jan Baptist van Helmont) verwendet, um das vitale Prinzip zu bezeichnen. Der Archeus reguliert den Stoffwechsel in Organismen (mit Sitz im Magen) und in der ganzen Natur. Obwohl z.B. van Helmont eine relativ ausgereifte Theorie der Verdauung aufstellt, wird die Theorie des Archeus aufgrund ihres Hintergrunds von den Vertretern der experimentellen Wissenschaften verworfen.

K2: Reids Rekonstruktion der Ideentheorie reicht nicht allein von Descartes bis Hume, sondern spannt den Bogen bis in die Antike zurück: „Doch von Plato bis Hume stimmen alle Philosophen darin überein, dass wir äußere Gegenstände nicht unmittelbar wahrnehmen und dass das unmittelbare Objekt der Wahrnehmung ein dem Geist gegenwärtiges Bild sein muss.“ (Essays 2.7, 263) Reid ist der Ansicht, dass die Ideentheorie seit rund 2000 Jahren stets durch die gleichen Vorurteile gestützt worden sei. Auch die „forms“ und „species“ der Aristoteliker und die „simulacra“ und „phantasms“ der Atomisten betrachtet Reid als Ausprägungen der Ideentheorie (vgl. Essays 4.2; Bd. 1, S. 433; Inquiry 7). Reids Kritik und ihre Ausweitung wird von einigen einflussreichen Philosophen des 20. Jh. wie Richard Rorty oder Martin Heidegger geteilt.

K3: Reid versteht seine Philosophie als Philosophie des Common Sense (vgl. Einleitung, Bd. 2). Reid ist jedoch nicht der erste Philosoph, der sich auf einen reichhaltigen Begriff des Common Sense stützt. Der französische Jesuit Claude Buffier (1661-1737) hatte in Traité des premières véritez (1724) bereits den Common Sense (sens comun) vom aristotelischen Gemeinsinn (sensus communis) unterschieden und eine eigenständige Philosophie des Common Sense entwickelt. Buffier beginnt, wie Descartes, mit einem ersten Prinzip der Erkenntnis, nämlich ,der unmittelbaren Empfindung [sentiment intime], die jeder von uns von seiner eigenen Existenz und davon hat, was er in sich vorfindet" (Buffier 1724, 9). Wenn wir jedoch allein dieses Prinzip für gewiss halten, sind uns viele Dinge zweifelhaft, z. B. dass es Körper gibt, dass wir nicht seit Ewigkeit existieren oder dass es andere denkende Wesen gibt. Doch diese Dinge halten wir faktisch für gewiss und an ihnen zu zweifeln ist lächerlich. Kein verständiger Mensch bezweifelt sie. Da aber diese Gewissheiten nicht aus der unmittelbaren Empfindung unserer eigenen Existenz folgen, müssen wir annehmen, dass es eine andere Regel der Wahrheit 
(rìgle de la vérité) gibt als die unmittelbare Empfindung unserer Existenz. Diese Regel bezeichnet Buffier als ,sens comun (sic! )“ und definiert sie als natürliche Disposition, Urteile über andere Gegenstände als über unsere unmittelbaren, bewussten Perzeptionen zu bilden, die nicht von vorgängigen Urteilen ableitbar sind (ebd., 25). Die Überschneidungen zwischen Reid und Buffier erschienen bereits den Zeitgenossen auffällig; der englische Übersetzer Buffiers witterte gar ein Plagiat, wie der Titel zeigt: First Truths, and the Origin of Our Opinions Explained, with an inquiry into the sentiments of moral philosophers relative to our primary notions of things to which is prefixed a detection of the Plagarism, Concealment and Ingratitude of Doctors Reid, Beattie, Oswald (London 1780). Reid setzt sich allerdings kritisch mit Buffier in Essays 6.7 auseinander (vgl. Maril-Lacoste 1982). Reids Philosophie ist es dann auch, die als Philosophie des Common Sense schulbildend geworden ist. Zur sogenannten schottischen Schule gehören etwa James Oswald (1727-1793), James Beattie (1735-1803), Dugald Stewart (1753-1828), Sir William Hamilton (1788-1856) oder James Frederick Ferrier (1808-1864); vgl. Broadie 2003. Reids Common-Sense-Philosophie wurde durch Pierre-Paul Royer-Collard (1763-1845, vgl. Royer-Collard 1913) und Victor Cousin (1792-1867; vgl. Cousin 1865, 540 ff.; Madden 1984) in Frankreich einflussreich, was sich unter anderem darin niederschlug, dass in Frankreich die erste Gesamtausgabe der Werke Reids entstand (Oeuvres complètes de Thomas Reid, hrsg. von Th. Jouffroy, Paris, 1828-1936; vgl. Madden und Manns 1987). Im 20. Jh. schließlich wurde die Philosophie des Common Sense durch zwei Autoren einflussreich, nämlich G. E. Moore und Roderick Chisholm (vgl. Lehrer 1976 und 1989, 6).

K4: Die Rede von einer „Anatomie der menschlichen Natur“ bzw. einer „Anatomie des Geistes" findet sich in Humes Treatise on Human Nature 1.4.6 bzw. 2.1.12. Mit Hume teilt Reid zwar die generelle Auffassung, dass es der Philosophie des Geistes noch nicht gelungen ist, zu einer Wissenschaft zu werden, doch lehnt Reid Humes Ansatz bei Ideen und Eindrücken entschieden ab (vgl. Wood 1994).

KS: Zu Reids Rekonstruktion der Ideentheorie vgl. einführend RoyerCollard 1913, 195-212; Lehrer 1989, Kap. 2; de Bary 2002, Kap. 6. Zur Vertiefung vgl. Daniels 1989, Kap. 4; Gallie 1989, Kap. 2. Wolterstorff 2001 rekonstruiert insgesamt sieben Vorwürfe Reids gegen die Ideentheorie (IT): (1) Es gibt keine (guten) Argumente zugunsten der IT (ebd., 65 ff.); (2) Die IT macht das Bewusstsein von äußeren Objekten zu einem Bewusstsein von inneren, geistigen Bildern (85ff.); (3) Die IT setzt voraus, dass Wahrnehmungsüberzeugungen aus Sinnesempfindun- 
gen abgeleitet werden (92 ff.); (4) Ideen als geistige Objekte und kausale oder assoziative Relationen zwischen ihnen können Wahrnehmung, Erinnerung und Denken nicht erklären (46 ff.); (5) Die IT erklärt die Tätigkeit von Personen wie Aktivität von Materie (54ff.); (6) Die IT nimmt an, dass Empfindungen ihren Objekten ähnlich sein müssen (86 ff.); (7) Die IT vermischt intentionale Wahrnehmungen und nicht-intentionale Empfindungen (80 ff.). - In diesem Abschnitt liegt das Augenmerk auf (1) bis (3), wobei (2) der „Unmittelbarkeitsthese“ und (3) der „Deduktionsthese" entspricht (vgl. Einleitung, Bd. 1, 11.1.2).

K6: Reids philosophiegeschichtliches Narrativ lautet wie folgt: Descartes hat der Philosophie mit dem Begriff der Idee einen neuen Weg gezeigt (den sog. „Way of Ideas“, eine Bezeichnung, die vermutlich aus Edward Stillingfleet „The Bishop of Worcester's Answer to Mr Locke's Letter" stammt; vgl. Stillingfleet 1987, 80). Dabei handle es sich freilich um einen Irrweg, dem Autoren wie Malebranche, Locke, Berkeley und Hume bis zu seinem unvermeidlichen Ende gefolgt sind, nämlich bis zum Skeptizismus. Die Ideen legen sich gleich einem Schleier zwischen den Geist und die Wirklichkeit materieller und von uns unabhängiger Objekte der Außenwelt. Als Konsequenz davon wird nicht nur fraglich, ob wir diese Wirklichkeit erkennen können, sondern auch, ob sie überhaupt existiert. Während sich Descartes oder Locke noch darum bemühten, über den Umweg der Ideen den Zugang zu dieser Wirklichkeit zu behaupten, glaubt Berkeley den drohenden Skeptizismus nur dadurch vermeiden zu können, dass er die Existenz dieser Wirklichkeit bestreitet. Berkeley habe gezeigt, dass „alles im Universum auf zwei Kategorien reduziert werden kann, nämlich geistige Wesen (minds) und Ideen im Geist" (Essays 2.10, 281). Humes Treatise, den Reid als Ausdruck eines destruktiven Skeptizismus versteht, geht diesen Weg konsequent zu Ende, indem er auch noch die Realität der Geister leugnet ,und in der Natur nichts übrig lässt als Ideen und Eindrücke, ohne ein Subjekt, dem sie eingedrückt werden könnten“ (Inquiry 1.5, 102). Humes Skeptizismus, so Reids Pointe, lässt nun aber mit aller Deutlichkeit hervortreten, was am Anfang diese Irrwegs gestanden und ihn erst ermöglicht hat: Ideen. Reid widmet der Analyse der Schriften der Ideentheoretiker vor allem in den Essays viel Raum und lässt sie auch selbst zu Wort kommen. Im Inquiry werden jedoch Arnauld und Leibniz nicht erwähnt und Malebranche entweder stets im Verbund mit Descartes und Locke oder nur beiläufig behandelt. Die Integrationen von Autoren wie Arnauld bereitet Reid in den Essays dann erdenkliche Mühe (Essays 2.13, 297/8; vgl. Nadler 1986). Malebranche und Leibniz werden als Spezialfälle behandelt 
(Essays 2.15, 309). Reids philosophiehistorisches Narrativ wurde bereits früh angefochten. Bereits Thomas Brown (1733-1820) meinte, dass alle Philosophen vor Reid (außer Berkeley und Malebranche), „, noch bevor sie auch nur ein einziges seiner [Reids] Argumente gehört hätten, zugeben würden, wären sie von ihm gefragt worden, dass ihre Ansichten über die Ideen exakt dieselben sind wie die seinigen" (Brown 1820, Bd. 1, 339). Zur Kritik an Reids Rekonstruktion der neuzeitlichen Ideentheorie vgl. Yolton 1984; für Interpretationen einzelner Autoren, die von einem durch Reid geprägten Verständnis abweicht, Perler 1996 (zu Descartes) oder Saporiti 2006 (zu Berkeley); für eine Verteidigung der Interpretationen Reids vgl. de Bary 2002, 105-129; für eine systematische Aneignung der Reidschen Rekonstruktion vgl. Willaschek 2003, 112-119.

K7: Anspielung auf Descartes' Erste Meditation (AT VII, 19). Descartes weist dort die Vorstellung zurück, dass der Meditierende ein Wahnsinniger sein könnte, der glaubt, er sei ein Kürbis oder aus Glas gemacht. Diese Annahme, die ein Äquivalent zum Vertrauen auf das rationale Vermögen darstellt, darf von den Zweifeln der Ersten Meditation nicht betroffen sein (vgl. Frankfurt 1970). Jenseits des ironischen Tonfalls, dessen sich Reid befleißigt, artikuliert diese Passage einen fundamentalen Unterschied zwischen der Descartesschen und der Reidschen Auffassung einer rationalen Person: Descartes zufolge ist eine Person rational, die sich ihrer Vernunft bedient, Reid zufolge ist eine Person rational, die den Grundsätzen des Common Sense vertraut. In Reids Augen kommt die einseitige Privilegierung der Vernunft einer Beschneidung der Grundsätze des Common Sense gleich und ist deshalb eine Form der Irrationalität (vgl. Reids Rede von einem „metaphysischen Wahnsinn“, Inquiry 7.4; Bd. 1, S. 423). Der ironische Tonfall ist nicht unangemessen, denn Reid zufolge begegnet man den Leugnern des Common Sense am besten mit der Waffe der Lächerlichkeit.

K8: Thomas Hobbes hat im Zuge seiner Einwände gegen Descartes' Zweite Meditation kritisiert, auf die eigene Existenz könnte ebenso gut aufgrund einer beliebigen Tätigkeit geschlossen werden, es müsse sich nicht um das Denken handeln: Ich spaziere; es ist unmöglich, dass ich spazieren gehe und nicht existiere; also existiere ich (vgl. AT VII, $195 \mathrm{ff}$.). Reid scheint hier Hobbes' Einwand aufzunehmen. Beide übersehen aber, wie zentral für Descartes' Argumentation der Bezug auf das Denken als generische und nicht-körperliche Tätigkeit ist.

K9: Ein Enthymem ist eine unvollständige Schlussform, nämlich ein um die Oberprämisse verkürzter Syllogismus. Descartes' Cogito-Argument könnte als Enthymem rekonstruiert werden, dem die Oberprä- 
misse „Alles, was denkt, existiert“ fehlt. Doch Descartes hält das „Ich denke, ich existiere" für unmittelbar gewiss und betrachtet es nicht als Ergebnis eines Schlusses.

K10: Entgegen der gängigen Auffassung, die Descartes und Locke als Rationalisten bzw. Empiristen kontrastiert, führt Locke der theoriegeschichtlichen Narration von Reid zufolge Descartes' ideentheoretischen Ansatz konsequent weiter. Von der Ideentheorie und ihrer Abkehr von den Grundsätzen des Common Sense wird nämlich in erster Linie auch die Auffassung des denkenden Subjekts, der Person, nachteilig beeinflusst. Subjekt und Person werden als Bewusstsein, als Träger von Ideen bestimmt. Reid zufolge kommt diese Entwicklung in Humes These, Personen seien nichts weiter als Bündel von Ideen, zu einem konsequenten Abschluss. Reid hat sich insbesondere in Essays 3.6 mit Lockes Theorie der personalen Identität auseinandergesetzt. Der zweiten Auflage seines Essays hat Locke das Kapitel „Of identity and diversity“ (Essay II, xxviii) hinzugefügt, in welchem er eine einflussreiche Theorie der personalen Identität (der Identität einer Person als dieselbe Person über die Zeit hinweg) vertritt.

K11: Nicht zuletzt aufgrund solcher Stellen ist Reids Common-Sense-Philosophie als Ausdruck eines Anti-Intellektualismus verstanden worden, wozu auch ein Strang der positiven Rezeption Reids beigetragen hat; vgl. etwa A. Schimberg, in: Royer-Collard 1913, xvii: „Das Haupt der schottischen Schule ist ein frommer anglikanischer Priester, der sich anschickt, aufgeschreckt durch das Wüten der Humeschen Philosophie und mit einem moralischen und religiösen Ziel vor Augen, den grundlegenden Spiritualismus zu retten und eine Philosophie auf unbestreitbaren und unerschütterlichen Sicherheiten aufzubauen, nämlich den Gegebenheiten des Common Sense." Einige Interpreten betrachten die Missverständnisse, die die Rezeption von Reid als einem Common-Sense-Philosophen hervorgerufen haben, und die Verworrenheit, mit der Reid selbst diesen Begriff behandelt, als guten Grund, ihn soweit es geht nicht mehr zu gebrauchen, vgl. Nichols 2007, 21: „Given the historiographical role of common sense in misinterpretations, students of Reid's work are faced with the choice of either explaining in detail just what he means by describing his theories as commonsensical, or in stating his theories in ways that minimally play upon his appeal to common sense." Reids Gebrauch des Ausdrucks „Common Sense“ ist bereits früh als unpräzise kritisiert worden, da in ihm, wie etwa Joseph Priestley es ausdrückte ,independent, arbitrary, instinctive principles“ bunt vermischt vorliegen. In den Prolegomena warf Kant Reid vor, er und andere schottische 
Philosophen des Common Sense hätten die skeptischen Herausforderungen Humes ganz missverstanden, ,indem sie immer das als zugestanden annahmen, was er gerade bezweifelte, dagegen aber mit Heftigkeit und mehrentheils mit großer Unbescheidenheit dasjenige bewiesen, was ihm zu bezweifeln niemals in den Sinn gekommen war" (AA. 4:258). Auch der Biograph und Common-Sense-Philosoph Dugald Stewart empfand den Ausdruck als vieldeutig und schlug die Bezeichnung „fundamental laws of human belief“" vor. Wolterstorff hält Reids Begriff für „not just confusing but confused“ $(2001,218)$ und beurteilt die Rezeption der Reidschen Philosophie unter diesem Etikett als ,its great misfortune“ (ebd., 215). Andere sind optimistischer, vgl. Marcil-Lacoste 1982; Schulthess 1983; Rysiew 2003; Pakaluk 2003.

K12: Juristischer Ausdruck aus dem angelsächsischen Common Law. Das ursprünglich aus dem Mittelalter stammende Quo warranto („Mit welcher Berechtigung?") wurde später zur Bezeichnung einer schriftlichen Aufforderung, die Rechtmäßigkeit der Ausübung amtlicher oder staatlicher Befugnisse auszuweisen. Quo-warranto-Verfahren spielten in der englischen Geschichte insbesondere im 16. Jh. im Vorgehen der Stuarts gegen Städte wie London eine Rolle (vgl. Hume, History of England, Bd. VI, Kap. 59).

K13: Bei dieser Aufzählung handelt es sich um ein und dieselbe Ähnlichkeitsthese: Materielle Objekte und Sinnesempfindungen dieser Objekte müssen sich ähnlich sein. Gegenüber der Ähnlichkeitsthese ist für Reid Folgendes entscheidend: Wenn eine Eigenschaft eines Objekts in unseren Sinnen eine Empfindung (sensation) verursacht, so hat diese Empfindung keinerlei Ähnlichkeit mit der Eigenschaft. Vielmehr veranlasst uns die Empfindung dazu, dass wir uns das Objekt als so und so beschaffen vorstellen (conceive), und zwar direkt, ohne bewusste Wahrnehmung der Empfindungen. Die Art und Weise dieser Veranlassung ist durch unsere natürliche Konstitution (durch die menschliche Natur) festgelegt (vgl. DeRose 1989). Reid vergleicht die Weise, in der wir Objekte wahrnehmen, mit dem Verstehen der Bedeutungen einer Sprache: Obschon Sprecher Worte verwenden, sind sich Hörer des Gesagten unmittelbar bewusst. Unsere Empfindungen sind einer natürlichen Sprache vergleichbar, die den Zweck hat, wirkliche Objekte zu bezeichnen (dieser Gedanke findet sich auch in Berkeleys An Essay Towards a New Theory of Vision SS 139 ff., Bd. 1, S. 358; vgl. Atherton 1990, 104-5. u. $195 \mathrm{ff}$.). Reids Zuschreibung einer Ähnlichkeitstheorie ist stark bis grob vereinfachend. Den Aristotelikern zufolge beinhaltet der Prozess der Wahrnehmung eine Angleichung oder Assimilation des Wahrnehmungs- 
subjekts an das Wahrnehmungsobjekt: das Subjekt nimmt die Form des Objekts auf und gleicht sich ihm so an. Die Ideentheoretiker bestreiten, dass Wahrnehmungsobjekte aus Materie und Form bestehen. Wenn es aber in den materiellen Gegenständen keine Formen gibt, kann sich das Subjekt nicht an Formen angleichen. An die Stelle einer Assimilation tritt eine Kausalrelation zwischen Objekten und Ideen. Ideen repräsentieren Objekte, doch sie müssen ihnen gerade nicht ähnlich sein. So behauptet Descartes keineswegs die Notwendigkeit einer Ähnlichkeit zwischen Ideen und ausgedehnten Körpern. Insbesondere die körperlichen sinnlichen Ideen (oder Bilder) müssen keine Ähnlichkeit mit den von ihnen repräsentierten materiellen Gegenständen aufweisen. Sie sind vielmehr eine Art kodierte Zeichen für jene Gegenstände, die kausal für ihr Auftreten verantwortlich sind (vgl. AT VI, 130; AT X, 413). Locke allerdings behauptet im Zuge seiner Unterscheidung zwischen sekundären Qualitäten (Farben, Gerüche, Töne usw.) und primären Qualitäten (Form, Größe, Bewegung usw.), dass zwar nicht sekundäre Qualitäten, wohl aber primäre Qualitäten den Ideen, die wir von ihnen haben, ähnlich sind (vgl. Essay II, viii, \15; Bd. 1, S. 275). Diese Ähnlichkeitsthese ist aber von Berkeley und Hume scharf kritisiert worden (vgl. Treatise 1.4.4).

K14: Diese Stelle ist wohl wie folgt zu verstehen: Reid ist der Ansicht, dass der Ideentheorie eine Ähnlichkeitsthese (vgl. K13) zugrunde liegt, für die die Ideentheoretiker nicht eigens argumentieren. Dieser These zufolge muss die materielle Welt unseren Empfindungen ähnlich sein und umgekehrt. Diese These hält Reid für völlig unbegründet, denn es ist offensichtlich, so Reid, dass sich eine Empfindung und ein Gegenstand in der Welt ganz und gar unähnlich sind. Er illustriert dies zunächst am klaren Fall einer Schmerzempfindung und einer Schwertspitze, die, so dürfen wir ergänzen, den Schmerz verursacht. Diesen offensichtlichen Sachverhalt versucht Reid nun per analogiam auf das Verhältnis von anderen Empfindungen und den kausal für sie verantwortlichen Eigenschaften von Gegenständen in der Welt zu übertragen: So wie der Schmerz der Schwertspitze ganz und gar unähnlich ist, seien auch unsere (Begriffe von) subjektiven Empfindungen von Ausdehnung oder Härte (unseren Begriffen von) Ausdehnung oder Härte als Eigenschaften von Gegenständen in der Außenwelt ganz und gar unähnlich. Zwar begegnen uns gewisse Eigenschaften der materiellen Gegenstände (wie etwa Härte) stets als empfundene Härte (wir spüren oder fühlen die Härte eines Objekts). Dies bedeutet jedoch nicht, dass die tastbaren Eigenschaften der Gegenstände und unsere Tastempfindungen einander ähnlich sein müssen. Auch funktionieren Begriffe für objektive Eigenschaf- 
ten (wie Härte oder Ausdehnung) nicht gleich wie Begriffe für unsere durch diese Eigenschaften verursachten Empfindungen. Dieser letzte Gedanke über unterschiedliche Begriffe wird von Reid ebenfalls angedeutet.

K15: Als „Peripatetiker“ werden die Schüler des Aristoteles bezeichnet. Ursprung dieser Bezeichnung ist der Ort, in dem Aristoteles und seine Nachfolger in Athen Unterricht erteilten, nämlich im Peripatos (Wandelhalle).

K16: 1739 ist das Erscheinungsjahr des ersten Bands von Humes Traktat über die menschliche Natur.

K17: Reid deutet Descartes so, dass lediglich das Cogito (der Grundsatz des Bewusstseins) selbst-evident ist, alles andere aber durch die Vernunft evident gemacht werden muss. Demgegenüber sind für Reid alle Grundsätze des Common Sense (vgl. Einleitung, Bd. II) selbst-evident. Worin besteht der Unterschied zwischen Selbstevidenz und Evidenz? Viele englischsprachige Autoren brauchen „evident“ im Sinne von: Es liegt auf der Hand, warum X so ist, wie es ist, und zwar aufgrund von etwas anderem als X. Das bedeutet, dass der Grund für X evident ist. Was selbst-evident ist, erhellt aus sich selbst und kann nicht aus etwas anderem, dessen Begründungskraft auf der Hand liegt, bewiesen werden.

K18: Reids Vorwurf an Descartes lautet also, dass dieser die Ausgangslage aufgrund einer unausgewiesenen Vorliebe für theoretische Einfachheit unzulässig auf einen einzigen Grundsatz reduziert. Für Reid ist Descartes deshalb ein „Semiskeptiker“, der einigen Grundsätzen und kognitiven Vermögen vertraut, anderen hingegen nicht (vgl. oben Einleitung, 11.1). Auf den ersten Blick erscheint es seltsam, Descartes als Skeptiker zu betrachten, ist er doch angetreten, den Skeptizismus zu bekämpfen. Diese Auffassung passt jedoch zu Reids These, dass die Skepsis der Ideentheorie von Beginn an innewohnt (vgl. Inquiry 1.7; Bd. 1, S. 407).

K19: Reid übernimmt aus aristotelischen und cartesianischen Logiklehrbüchern der Zeit die Dreiteilung in Vorstellung (conception, concevoir, apprehensio), Urteil (judgement, jugements, iudicium) und Schluss (reasoning, raisonnement, ratiocinatio). Eine Vorstellung oder Apprehension ist etwa der Logik von Port Royal zufolge die Bildung oder Wahrnehmung einer Idee, ein Urteil das Verbinden mehrer Ideen, ein Schluss die Verbindung mehrer Urteile. Eine „apprehensio“ ist das geistige Erfassen eines Vorstellungsinhalts oder eines Dings. Es handelt sich um eine bloße Vorstellung der Dinge, ohne Beurteilung der Wahrheit des Vorgestellten. Ein einfaches Erfassen ist entsprechend die Erfassung eines einfachen Vorstellungsinhalts oder eines Dings simpliciter. So kann ich einen Apfel sehen, 
einen Kuckuck hören, an Gott denken oder mir einen Kuchen vorstellen. Mit dem Urteil meint Reid (wie die Formulierung „Urteil und Glaube“ zu verstehen gibt) weniger die Zusammensetzung zweier Vorstellungsinhalte zu einem Urteil als vielmehr den Glauben an die Existenz des aufgefassten Dinges. Einen Apfel sehen oder einen Kuckuck hören heißt urteilen oder glauben, dass ein Apfel bzw. ein Kuckuck existiert, hingegen heißt an Gott denken oder einen Kuchen vorstellen nicht urteilen oder glauben, dass Gott oder der Kuchen existieren. Aus diesem Grund gehören in der Sinneswahrnehmung Glaube oder Urteil und Vorstellung oder Erfassung stets zusammen.

K20: Locke zufolge stammen alle Ideen aus der Sinnesempfindung (sensation) oder aus der Reflexion (reflection), denn unser Geist befasst sich entweder mit äußeren sinnlichen Gegenständen oder mit den inneren Tätigkeiten des Geistes. Locke behauptet, dass es sich hierbei um die beiden einzigen Quellen für Ideen handelt (Essay II, i, \2; Bd. 1, S. 266).

K21: Locke definiert Wissen als die Wahrnehmung des Zusammenhangs und der Übereinstimmung oder der Nichtübereinstimmung und des Widerstreits irgendwelcher unserer Ideen (Essay IV, i, \2; Bd. 1, S. 288).

K22: Reid bezieht sich hier auf Inquiry 2.3-5, vgl. Essays 2.5; vgl. den systematischen Essay (11.3).

K23: Reid bezieht sich hier auf die Grundsätze des Common Sense, vgl. oben Einleitung, 11.1.

\subsubsection{Auszüge aus Essays on the Intellectual Powers of Man / Versuche über die intellektuellen Vermögen des Menschen (1785) (zitiert als: Essays)}

K24: Die Formulierung, die Reid hier Locke zuschreibt („Ideas are nothing but the immediate objects of the mind in thinking"), findet sich so nicht wörtlich bei Locke. Allerdings kann man inhaltlich ähnliche Formulierungen in dessen Essay Concerning Human Understanding finden. Dort heißt es z. B. in I, i, $\ 8$ auf die Frage, was eine Idee sei: „das ..., was auch immer der Gegenstand des Verstandes ist, wenn ein Mensch denkt" (Vgl. dazu die entsprechende Textstelle im Kapitel über Locke, Bd. 1, S. 264, und den dazugehörigen Kommentar K1). Im offenen Brief an Edward Stillingfleet, Bischof von Worcester, findet sich zudem fast wörtlich dieselbe Formulierung: „The things signified by ideas are nothing but the immediate objects of our minds in thinking" (John Locke, $A$ Letter to the Bishop of Worcester, 1697). 
K25: Johann Jakob Brucker (* 22. Januar 1696, Augsburg, † 26. November 1770, ebd.) war evangelischer Theologe und Verfasser einer Philosophiegeschichte. Reid spielt hier wohl auf seine Schrift Historia Philosophicae Doctrinae de Ideis (Augsburg 1723) an.

K26: Erst im 5. Essay („Of Abstraction“) nimmt Reid diesen Faden wieder auf. In Kapitel 5 dieses Essays („Observations Concerning the Names Given to Our General Notions") macht er deutlich, dass er selbst unter Allgemeinbegriffen (general notions) oder Allgemeinvorstellungen (general conceptions) „,immer die vorgestellten Dinge, und nicht den geistigen Akt des Vorstellens“ (Essays 5.5, 404) versteht. Und er fährt fort: „Die Pythagoräer und Platoniker haben ausschließlich diese allgemeinen Objekte der Vorstellung als Ideen bezeichnet. Da wir das Wort Idee von ihnen entlehnt haben, das nunmehr in allen Sprachen Europas bekannt ist, hielte ich es für das Richtige, wenn wir auch dessen Bedeutung entlehnen und es nur dazu verwenden würden, uns auf das zu beziehen, was auch sie damit meinten. Ich gehe davon aus, dass wir ein Wort ohne Mehrdeutigkeit brauchen, das die Dinge, die wir ausschließlich vorstellen, von den Dingen unterscheidet, die existieren. Würde das Wort Idee ausschließlich zu diesem Zweck verwendet, hätten wir es in seiner ursprünglichen Bedeutung wiederhergestellt und diesem Bedürfnis Rechnung getragen. Selbstverständlich können wir mit den Platonikern hinsichtlich der Bedeutung des Wortes Idee übereinstimmen, ohne deren Theorie bezüglich der Ideen zu übernehmen“ (ebd.).

K27: Die folgende Diskussion betrifft das Problem, das wir heute als Problem der intentionalen Inexistenz bezeichnen würden. Reid bestreitet vehement, dass wir ein unmittelbar gegenwärtiges Objekt unserer Vorstellung benötigen, wenn wir uns auf etwas intentional beziehen (d.h. vermittels einer Vorstellung), das nicht existiert oder existiert hat. Dass eine derartige Argumentation erfolgreich ist, ist eines der zentralen Fundamente einer Alternativkonzeption zum ideentheoretischen Paradigma. Denn gerade der elegante Umgang mit der intentionalen Inexistenz scheint einer der systematischen Vorteile der Ideentheorie zu sein, die ja mit den Ideen genau solche unmittelbaren Objekte zur Verfügung stellt. Reids Antwort scheint nur vor dem Hintergrund einer adverbialen Analyse auch des Vorstellungsbegriffs überzeugend, die aber ihrerseits Probleme aufwirft. Vgl. dazu den systematischen Essay (11.3), unten S. 445 f. Außerdem zum Thema: Nichols 2007, Kap. 2 \& 7.

K28: Die meisten Aristoteliker des Mittelalters gingen davon aus, dass es vermittelnde Entitäten geben muss, damit eine Wahrnehmungsrelation zwischen zwei getrennten Dingen entsteht. Dieser Wahrnehmungstheorie zufolge werden Eigenschaften materieller Objekte auf das wahr- 
nehmende Subjekt übertragen, und zwar mithilfe besonderer Entitäten, sog. ,species in medio“. Die ,species in medio“ vermitteln über ein Medium (Luft, Licht) zwischen Objekten und ihrer Wahrnehmung. Erblickt ein Betrachter ein Objekt, nimmt er dessen wahrnehmbaren Eigenschaften auf und bildet auf dieser Grundlage Vorstellungsbilder (species sensibilis), die der Intellekt in Verstandesbegriffe (species intelligibilis) umformt. So werden nicht nur Vorstellungsbilder, sondern die Form von Objekten erfasst. In der Wahrnehmung aufgenommen wird jedoch nicht die Materie, sondern nur die Form dieser Eigenschaften. Wer etwa eine Farbe sieht, nimmt nur das auf, was für die Struktur der Farbe verantwortlich ist: ein immaterielles Prinzip. Sobald dieser Prozess des Aufnehmens abgeschlossen ist, befindet sich die Form der wahrnehmbaren Eigenschaft im Wahrnehmenden. Wahrnehmung ist passiv: Der Wahrnehmende „erleidet“ etwas, wenn die Formen der wahrnehmbaren Eigenschaften auf ihn übertragen werden, und er gleicht sich diesen Eigenschaften an, ohne dazu eine besondere Leistung erbringen zu müssen (vgl. Spruit 1994; Pasnau 1997). Dieser Theorie stellt sich die Frage, was ein Betrachter sieht: das Objekt selbst oder nur eine Species? Einige Autoren sind der Ansicht, dass die Species ein Bild von der Sache sei, nicht die Sache selbst. Aus diesem Grund rechnet Reid der Ideentheorie auch die Theorie der Species und der Formen zu.

\subsection{Essay: Empfindung und Wahrnehmung - Reids Adverbialismus}

Der Versuch einer konsequenten Unterscheidung zwischen Empfindung und Wahrnehmung nimmt gleichermaßen einen zentralen Platz in Reids Kritik an der Ideentheorie wie in seiner eigenen, als Alternative zur Ideentheorie entwickelten Theorie der Wahrnehmung ein. Im Inquiry von 1764 heißt es dazu programmatisch:

Empfindung und die Wahrnehmung äußerer Gegenstände durch die Sinne sind gemeinhin für ein und dasselbe gehalten worden, obwohl sie ihrer Natur nach sehr verschieden sind. Die Zwecke des alltäglichen Lebens machen eine solche Unterscheidung nicht erforderlich und die überkommenen Meinungen der Philosophen neigen dazu, sie durcheinander zu bringen. Doch es ist unmöglich, eine angemessene Auffassung der Operationen unserer Sinne zu erlangen, ohne sorgfältig auf diese Unterscheidung zu achten. (Inquiry 6:20, 183)

Um die Leistungsfähigkeit, aber auch die Grenzen dieser Unterscheidung soll es im Folgenden gehen. 


\subsubsection{Adverbialismus}

Ausgangspunkt der Untersuchung ist Reids Analyse der Empfindungen. Empfindungen sind für Reid Zustände des Bewusstseins und damit Zustände des Geistes. Doch Empfindungen sind keine intentionalen Zustände, sondern müssen von diesen scharf abgegrenzt werden. Der Grund dafür ist, dass Empfindungen kein intentionales Objekt haben. Daraus ergibt sich natürlich unmittelbar, dass in Reids Konzeption des Geistes die Brentano'sche These nicht gilt: Nicht alle geistigen Zustände sind intentionale Zustände in Brentanos Sinne. ${ }^{10}$

In seinem Inquiry von 1764 bringt Thomas Reid diese Analyse folgendermaßen auf den Punkt:

Die Form des Ausdrucks ,Ich empfinde Schmerzen' scheint zu implizieren, dass es sich bei der Empfindung um etwas von dem empfundenen Schmerz Verschiedenes handelt. Tatsächlich gibt es aber keinen derartigen Unterschied. Genau so wie ,einen Gedanken denken' ein Ausdruck ist, der nichts anderes bezeichnet als ,denken', bezeichnet ,einen Schmerz empfinden' [feeling a pain] nichts anders als schmerzen' [being pained]. Was wir über Schmerzen gesagt haben, kann auf jede andere bloße Empfindung angewendet werden. (Inquiry 4:20, 183)

Was Reid in dieser kurzen Passage skizziert, ist eine adverbialistische Analyse von Empfindungsausdrücken, wie wir sie in der Zuschreibung von Empfindungen verwenden, und - obwohl die Unterscheidung zwischen sprachlicher Ebene und Objektebene auch bei Reid, wie bei allen anderen Autoren der frühen Neuzeit, in der Regel nicht hinreichend beachtet wird - auch eine adverbialistische Theorie der Empfindungen selbst.

Was macht eine Analyse geistiger Zustände adverbialistisch? Adverbialistisch ist eine Analyse mentaler Zustände, wenn sie mentale $\mathrm{Zu}$ stände der Art

A $\varphi-t$,

(wobei A das Subjekt des Zustands $\varphi$ ist) nicht etwa relational analysiert als

ARo,

(wobei $\mathrm{R}$ eine Beziehung bezeichnet und o das Objekt dieser Beziehung), sondern As $\varphi$-en vielmehr als adverbiale Kennzeichnung eines Zustands von A auffasst, also als

${ }^{10}$ Vgl. Brentano ${ }^{21924 . ~ Z u m ~ V e r h a ̈ l t n i s ~ v o n ~ R e i d s ~ T h e o r i e ~ z u ~ d i e s e r ~ T h e s e ~ v g l . ~ L e h-~}$ rer 1976, 15; Nichols 2007, 67. 
A $\varphi$-t in der $[\mathrm{o}]$-Weise

analysiert.

So wird in Reids Beispiel

A empfindet Schmerz

nicht als

A steht in einer Beziehung zu Schmerz

analysiert, sondern als

A schmerzt es,

was - da „es“ hier kein Objekt hat, sondern so funktioniert wie das „es“ in ,es regnet“ -, auch ausgedrückt werden kann als

A empfindet schmerzlich.

In diesem Ausdruck wird der adverbiale Charakter dieser Analyse besonders schön deutlich: „schmerzlich“ fungiert hier als adverbiale Modifikation des Verbums „empfinden“.

Der Adverbialismus kann sowohl als ontologische wie auch als semantische These vertreten werden. Der Grund für diese Unterscheidung ist, dass wir bei geistigen Zuständen zwischen ihrem Inhalt und ihrem ontologischen Status unterscheiden können: Adverbialismus kann nun entweder eine Theorie über den Inhalt geistiger Zustände sein oder eine Theorie über ihren ontologischen Status - oder beides zusammen. Als ontologische These besagt der Adverbialismus, dass (bestimmte) geistige Zustände nichts anderes sind als Modifikationen des Subjekts dieser geistigen Zustände. Als semantische These besagt er, dass der Inhalt (bestimmter) geistiger Zustände vollständig adverbial $\mathrm{zu}$ analysieren ist - unabhängig davon, was letztlich die Natur dieser geistigen Zustände ist.

Reid will nun in seiner Analyse der Empfindungen offenbar beide Thesen vertreten und systematisch zueinander in Beziehung setzen. So heißt es an einer Stelle in den Essays von 1785 (dem Werk also, das üblicherweise als ausgereiftere Darstellung von Reids Theorie betrachtet wird):

Der angenehme Geruch [einer Rose] ist, für sich genommen, [...] nichts anderes als eine Empfindung. Sie affiziert den Geist in bestimmter Weise; und diese Affektion kann man zum Gegenstand der Vorstellung machen [may be conceived], ohne den Gedanken an eine Rose oder an irgendeinen anderen Gegenstand. Die Empfindung ist nichts anderes als das, was zu sein man sie empfindet [it is felt to be]. Ihr Wesen besteht im Empfundenwerden; und wenn sie nicht empfunden wird, ist sie nicht. Es gibt keinen Unterschied zwischen der Empfindung und ihrem Empfinden. Das ist der Grund dafür, [...] dass in der Empfindung kein Gegenstand unabhängig von dem geistigen Akt existiert, durch den sie empfunden wird. (Essays 2.16, 310) 
Auf Reids Auffassung von Affektion in diesem Zusammenhang müssen wir weiter unten noch eingehen. ${ }^{11}$ Für den Moment reicht es festzuhalten, dass der Inhalt einer Empfindung, hier der Geruch der Rose, offenbar adverbialistisch zu analysieren ist: Das Wesen der Empfindung ist das Empfundenwerden in einer bestimmten Weise, das in keiner Weise auf einen Gegenstand gerichtet ist. Empfindungen, so können wir diesem Zitat weiterhin entnehmen, sind bloße Modifikationen des Geistes, weil ihr Inhalt adverbial analysiert werden kann. Der semantische Adverbialismus stützt bei Reid also den ontologischen argumentativ.

Dieser Zusammenhang ist allerdings keineswegs zwingend: Eine alternative Konzeption könnte sich die semantische These zu eigen machen, aber dennoch die ontologische These ablehnen - und umgekehrt. Der Grund dafür ist, dass die Analyse des Inhalts geistiger Zustände begrifflich unabhängig ist vom ontologischen Status dieser Zustände. ${ }^{12}$

Worin liegt nun Reids Ansicht nach der besondere Nutzen einer derartigen Analyse? Die Art und Weise, in der wir uns häufig sprachlich auf unsere Empfindungen beziehen, ist, wie aus dem Zitat aus dem Inquiry hervorgeht, mit dem wir diese Ausführungen begannen, irreführend: Sie legt nahe, dass wir bei Empfindungen zwischen einem Objekt der Empfindung und der Empfindung selbst in ähnlicher Weise unterscheiden können, wie wir es unproblematischer Weise im Falle intentionaler Zustände wie Gedanken, Überzeugungen, Wünschen, Hoffnungen etc. zu tun gewohnt sind, indem wir zwischen dem intentionalen Zustand und dem Objekt unterscheiden, auf das sich dieser Zustand kraft seines Inhalts bezieht.

Irreführend, so Reid, ist diese Ausdrucksweise deshalb, weil sie die tatsächliche Natur der Empfindungen verschleiert: Empfindungen mögen mentale Zustände sein, intentionale Zustände sind sie jedoch keinesfalls. Ihnen fehlt dazu etwas für intentionale Zustände Wesentliches, nämlich ein (sei es auch möglicherweise nichtexistentes) Bezugsobjekt,

11 Vgl. unten S. 460.

12 So vertreten Ducasse und Chisholm den Adverbialismus ausschließlich als semantische bzw. epistemologische These, Sellars ausschließlich als ontologische These. Vgl. Ducasse 1942; Chisholm 1957; Sellars 1975. Es ist deshalb problematisch, wenn man, wie Pappas 1989, Reid einmal einen Adverbialismus à la Ducasse und Chisholm unterstellt und später auf die enge Verwandtschaft zwischen Reids und Sellars' Konzeption von Empfindung verweist. Vgl. Pappas 1989, 162 und 164 Fn. 11. 
das sie qua geistiger Zustand haben ${ }^{13}$. Eine adverbialistische Analyse von Empfindungsausdrücken wird diesem besonderen Charakter von Empfindungen gerecht, da diese Analyse nicht erst den Eindruck erweckt, wir könnten die Empfindung von dem Empfinden der Empfindung in ähnlicher Weise trennen wie das Bezugsobjekt eines Gedankens vom Denken dieses Gedankens.

Denn das, so Reid, ist nicht richtig: Die Empfindung verhält sich zum Empfinden der Empfindung nicht so wie der Gedanke zum Denken eines Inhalts, sondern so wie ein Gedanke zum Denken des Gedankens. Genau wie das Denken des Gedankens, dass ich bin, nichts anderes ist als der Zustand des Denkens, dass ich bin, so ist das Empfinden einer Schmerzempfindung nichts anderes als der Zustand des Schmerzempfindens, d.h. die Schmerzempfindung selbst.

Gerade die Analogie zwischen Empfindungen und Gedanken, die Reid in seiner Überlegung so geschickt einsetzt, legt allerdings auch nahe, dass man unter dieser Voraussetzung streng genommen auch intentionale $\mathrm{Zu}-$ stände wie Gedanken oder Überzeugungen adverbial analysieren sollte: Auch für Gedanken gilt ja, dass sie qua Gedanken erst einmal Zustandsweisen des denkenden Subjekts sind. Hinsichtlich ihrer ontologischen Kategorisierung scheinen sich Gedanken also ganz genauso zu verhalten wie Empfindungen - beide sind Modifikationen eines Subjekts, d.h. in Reids dualistischem Weltbild: eines Subjekts, sofern es Geist ist. ${ }^{14}$

Doch wir können, wie bereits erwähnt, bei geistigen Zuständen zwischen zwei Betrachtungsweisen unterscheiden: wir können einen geistigen Zustand qua Zustand betrachten und wir können ihn qua Inhalt betrachten. Im Falle der Empfindungen ändert sich durch diesen Wechsel der Betrachtungsweise nichts: Eine Empfindung ist qua Zustand nach Reid genau die Empfindung, die sie qua Inhalt ist. So ist eine Schmerzempfindung nichts anderes als das schmerzliche Empfinden. Der Inhalt der Empfindung ist, mit anderen Worten, die Art und Weise des Empfindens selbst.

Dasselbe gilt nun aber nicht für Gedanken: das Denken eines Gedankens qua Zustandsweise ist vom Denken eines Gedankens qua Inhalt ver-

13 Diese Qualifikation ist nötig, da wir sehen werden, dass Empfindungen bei Reid in anderer Weise durchaus ein Bezugsobjekt haben: Sie fungieren als natürliche Zeichen für Eigenschaften von Gegenständen. Dieses Bezugsobjekt macht die Empfindungen, wie sich zeigen wird, allerdings nicht selbst intentional, da dieses Bezugsobjekt nicht ihr intentionales Objekt ist, sondern das intentionale Objekt eines anderen geistigen Zustands. Vgl. dazu unten S. 451.

14 Vgl. Essays 1.2. 
schieden. Inhalte von Gedanken sind nichts anderes als intentionale Objekte, wobei der Ausdruck „Objekt“ hier in einem sehr weiten Sinne verwendet wird.

Dieser Umstand veranlasst Reid nun nicht etwa dazu, die adverbialistische Ontologie für Gedanken ohne weiteres abzulehnen, wohl aber dazu eine entscheidende semantische Disanalogie zwischen Empfindungen und Gedanken zu entwickeln, die auch die ontologische Analogie beeinträchtigt.

Für diese semantische Disanalogie und ihre ontologischen Auswirkungen argumentiert Reid wiederum anhand der Analyse unserer alltäglichen Redeweise. Zunächst finden wir hier wieder die Feststellung einer oberflächlichen Ähnlichkeit der grammatischen Struktur, von der wir bereits wissen, das Reid sie letztlich ablehnt:

So etwa ,Ich empfinde einen Schmerz'; ,Ich sehe einen Baum‘: Der erste Satz bezeichnet eine Empfindung, der zweite eine Wahrnehmung. Die grammatische Analyse beider Ausdrücke ist dieselbe: denn beide bestehen aus einem aktiven Verb und einem Objekt. Wenn wir uns aber den Dingen zuwenden, die von diesen Ausdrücken bezeichnet werden, so werden wir feststellen, dass im ersten die Unterscheidung zwischen Handlung [act] und Gegenstand [object] nicht real, sondern bloß grammatisch ist, während sie im zweiten nicht bloß grammatisch ist, sondern real. (Inquiry 6:20, 182-3)

Wenn wir davon ausgehen, dass Wahrnehmungen für Reid (zumindest auch) Gedanken sind, dann finden wir in diesem Zitat also einmal mehr die Begründung einer ontologische These auf der Basis einer semantischen Analyse unserer alltäglichen Redeweise und deren epistemologischer Interpretation: Die ontologische These ist nunmehr, dass das Sein eines Gedankens sich nicht in seinem Zustandsein erschöpfen kann. Er ist mehr als bloße geistige Tätigkeit; er ist eine Tätigkeit, die sich wesentlich auf ein Objekt richtet.

Die Begründung für diese These ist, dass dieses Zustandsein für sich genommen nicht erklären kann, in welchem Sinne Gedanken ein intentionales Objekt haben. Und diese Begründung ist ihrerseits nichts anderes als die philosophische Interpretation der Analyse unserer alltäglichen Redeweise: Die Erklärung dafür, dass unsere Gedanken wesentlich ein intentionales Objekt haben, ist nötig, weil sich der Ausdruck der Gedanken - im Gegensatz zu unserem Sprechen über Empfindungen - nicht restlos adverbial analysieren lässt. ${ }^{15}$

15 Dass diese Disanalogie zumindest umstritten ist, zeigen die adverbialistischen Analysevorschläge von intentionalen geistigen Zuständen durch Sellars 1975 und Tye 1984. 
Mit dieser Begründung der ontologischen These bleiben Gedanken zwar qua geistige Zustände einer adverbialen Beschreibung zugänglich; allerdings ist diese Beschreibung auch als ontologische Charakterisierung des Denkens nun nicht mehr vollständig: Sie charakterisiert Gedanken bloß als Zustände und vernachlässigt die Inhaltsdimension, die sie erst zu den Gedanken macht, die sie jeweils sind.

Der Grund für die skizzierte semantische Disanalogie und ihre ontologischen Auswirkungen ist also darin zu sehen, dass Reid einen engen Zusammenhang zwischen der Analyse der alltäglichen Redweise über geistige Zustände und ihrer ontologischen Interpretation sieht. Dieser Zusammenhang ist allerdings alles andere als selbstverständlich. Wieso also glaubt Reid, dennoch so argumentieren zu können?

An dieser Stelle muss man sich an die Rolle erinnern, die Reid dem Common Sense zuschreibt: Den Grundsätzen des Common Sense kommt, wie wir gesehen haben, die Rolle quasi-axiomatischer Konstanten unseres Denkens und Handelns zu. Und welche Grundsätze tatsächlich die Dignität solcher Konstanten haben, hängt wiederum davon ab, welche Konstanten im System unserer alltäglichen Überzeugungen eine unverrückbare Rolle spielen.

Welche aber sind das? Einen wichtigen Wegweiser kann hier die Analyse unserer alltagssprachlichen Redeweise darstellen. Doch dabei muss man äußerst vorsichtig vorgehen, da uns die Oberflächengrammatik in die Irre führen kann, wie das Beispiel der Empfindungen zeigt: Was scheinbar in geistige Tätigkeit und ihren (gedachten) Gegenstand zerfällt, ist, wenn Reid recht hat, in Wirklichkeit nichts anderes als bloße, gegenstandslose geistige Tätigkeit.

Wie kann uns vor dem Hintergrund solcher Täuschungsfälle die Alltagssprache noch als Wegweiser der philosophischen Analyse dienen? Nun, beispielsweise, indem man sich an genau diejenigen sprachlichen Verwendungsweisen hält, die sich durch eine alternative Analyse unseres Sprechens nicht weganalysieren lassen. Denn im Normalfall ist der Sprachgebrauch Reids Ansicht nach tatsächlich ein wichtiger Indikator für eine am Common Sense orientierte Analyse ${ }^{16}$ - allerdings nur dann, wenn nicht auf einen alternativen Sprachgebrauch verwiesen werden kann, der die fragliche Implikation nicht nahe legt. Genau von dieser Art, so argumentiert Reid, sind aber unsere Auffassungen über die Common

16 Vgl. Einleitung 11.1. 
Sense-Analyse intentionaler geistiger Zustände - und das unterscheidet sie eben von der Analyse unserer Empfindungszuschreibungen.

Dieser Umstand liefert uns also zunächst den entscheidenden Beleg für eine im Common Sense verankerte Unterscheidung der Analyse des Inhalts von Empfindungen und des Inhalts intentionaler Zustände. Denn, so kann Reid argumentieren, wir können intentionale Zustände tatsächlich nicht ohne Verweis auf ihr (intentionales) Objekt vollständig charakterisieren; für Empfindungen ist das, wie wir gesehen haben, möglich. Diese semantische Analyse hat aber, so können wir vor dem Hintergrund der Reidschen Methodologie des Common Sense nun feststellen, auch eine ontologische Dimension: Das Sein von Empfindungen erschöpft sich also tatsächlich im Empfundenwerden; das Sein von intentionalen Zuständen hingegen besteht in mehr als dem Gedachtwerden auch wenn dies ein wesentlicher Teil dieses Seins ist. Das Sein der Gedanken besteht darüber hinaus im Bezug auf ein (intentionales) Objekt des Gedankens - und unabhängig von dem Bezug auf dieses Objekt ist der Gedanke eben nicht vollständig charakterisierbar und damit in seinem Wesen nicht vollständig erfasst.

Die adverbiale Analyse ist im Falle intentionaler Zustände also nach Maßgabe von Reids Common Sense Theorie nicht nur semantisch abzulehnen, sondern auch ontologisch unvollständig, weil sie die (vorgeblichen) relationalen Eigenschaften nicht mit einbezieht, die die intentionalen Zustände kraft ihres intentionalen Objekts erst zu den Zuständen machen, die sie sind.

An dieser Stelle entsteht allerdings ein Problem für Reids Disanalogie, da die intentionale Bezugnahme nicht erfolgreich sein muss, damit der intentionale Zustand zu dem Zustand wird, der er kraft seines Inhalts ist. Sofern wir etwas wahrnehmen, hat ein intentionaler Zustand zwar ein reales Objekt: Wahrnehmen ist ein Erfolgsverb ${ }^{17}$; dennoch bleiben Probleme für Fälle wie Halluzination, Träume, Produkte der Einbildung oder Erinnerungen, in denen unser intentionaler Zustand kein unmittelbar gegenwärtiges Objekt zu haben scheint.

Reid legt viel Wert darauf, dass die Objekte unserer intentionalen $\mathrm{Zu}$ stände nicht existieren müssen, damit wir uns intentional auf sie beziehen können. Dieser Punkt ist ihm sogar ausgesprochen wichtig, denn er stellt einen Grundpfeiler seiner Kritik an der Ideentheorie dar: ${ }^{18}$ Eine wesentliches Argument der Ideentheoretiker, so wie Reid sie verstand,

17 Vgl. dazu Austin 1962, 85-104.

18 Vgl. dazu Einleitung Bd.1, 11.1.2. 
war gerade das angebliche Vorhandensein eines Stellvertreterobjekts in Fällen, in denen kein irgendwie gegenwärtiges Objekt als intentionales Objekt zur Verfügung steht. Die Ideen waren genau solche Stellvertreterobjekte: Sie sind die unmittelbaren Objekte der intentionalen Bezugnahme, da sie auch dann existieren, wenn ihnen kein reales Objekt entspricht, während letztere nur als unmittelbare Objekte intentionalen Bezugs fungieren.

Reid greift diese Auffassung vehement an, ${ }^{19}$ indem er insistiert, dass das intentionale Objekt geistiger Zustände immer der Gegenstand ist, auf den sie sich kraft ihres Inhalts unmittelbar beziehen - und dieser Gegenstand existiert zwar in vielen Fällen, in vielen anderen existiert er jedoch nicht (häufig ohne dass wir als Subjekte dieser intentionalen Zustände uns dessen bewusst sind). So funktionieren eben intentionale Zustände. Dass wir uns häufig auf Dinge intentional beziehen, die nicht existieren, ist, mit anderen Worten, eine nicht weiter erklärbare oder auch nur erklärungsbedürftige Grundeigenschaft unserer intentionalen Bezugnahme.

Man mag das für überzeugend halten oder nicht: Problematisch ist dieser Aspekt seiner Analyse auf jeden Fall für die von ihm vertretene Disanalogie zwischen Empfindungen und intentionalen Zuständen, die wir diskutiert haben. Denn diese Auffassung legt nahe, dass sich intentionale Zustände eben doch vollständig und eindeutig ganz aus sich selbst heraus charakterisieren lassen, ohne die Einbeziehung relationaler Eigenschaften. Denn der intentionale Bezug ist dann nicht wesentlich ein erfolgreicher Bezug - und damit überhaupt nicht wesentlich ein realer Berug. Als nicht wesentlich reale Bezugnabme handelt es sich dann aber nur um eine Tätigkeit oder Handlung des Geistes - eine Handlung, die eben nicht mehr ,real“ (Inquiry 6.20, 183) unterschieden ist von ihrem Gegenstand. Und diese Tätigkeit sollte sich doch ebenso vollständig adverbial charakterisieren lassen wie andere bloße Tätigkeiten des Geistes auch. Und damit eröffnet sich einer vollständigen adverbialen Analyse wieder eine vielversprechende Perspektive.

19 Vgl. vor allem Essays 4.2; Bd. 1, S. 427. 


\subsubsection{Die Natur der Empfindung}

Empfindungen sind, soviel wurde bisher deutlich, für Reid geistige Zustände, die kein intentionales Objekt haben. Anders gesagt: Ihr Sein erschöpft sich im Empfundenwerden, ihr esse ist sentiri. ${ }^{20}$

Das heißt aber nicht, dass es in Reids Konzeption keinerlei Zusammenhang gäbe zwischen einer Empfindung und dem wahrgenommenen Gegenstand, der kausal für diese Empfindung verantwortlich ist: Empfindungen fungieren bei Reid nämlich als Zeichen für Eigenschaften von Gegenständen. ${ }^{21}$ Auf diese Weise spielen sie ihre (in der Regel) unentbehrliche Rolle in der Wahrnehmung, mit der wir uns im Weiteren noch beschäftigen werden. An dieser Stelle ist zunächst nur wichtig, dass jede einzelne Empfindung vermittels einer natürlichen, d.h. hier rein kausalen, Zeichenrelation genau eine Eigenschaft bezeichnet. ${ }^{22} \mathrm{Kraft}$ dieses natürlichen Zeichens bilden wir dann intentionale Vorstellungen (conceptions) dieser Eigenschaften, die wir in komplexen Wahrnehmungen von Gegenständen integrieren.

Dass Empfindungen als natürliche Zeichen fungieren, macht sie nicht selbst zu intentionalen Zuständen: Empfindungen werden vielmehr, in einem näher zu bestimmenden Sinne, als Zeichen für Eigenschaften verwendet. Diese Verwendung ist im Großen und Ganzen so automatisiert, dass wir uns ihrer nicht bewusst sind. ${ }^{23} \mathrm{Zu}$ einem erheblichen Teil müssen wir sie wohl sogar als angeboren auffassen. Empfindungen, die wir als Zeichen verwenden, ,schlagen uns [Eigenschaften] vor“" (Inquiry $2.8,111)$ - manche nur auf Grund unserer Erfahrungen und der Gewohnheit, die durch diese Erfahrungen erworben wurde, andere aber auch auf eine natürliche Weise (natural suggestions). Für die Verbindung

20 Vgl. Staudacher 2008. Dieselbe Formulierung findet sich auch bei Sellars $1982 \mathrm{mit}$ Bezug auf seine eigene Theorie. Wir werden auf die Frage zurückkommen, ob Reid eine vergleichbar klare Abgrenzung der Empfindung von intentionalen Zuständen gelingt.

21 Vgl. dazu ausführlich den hilfreichen Essay Staudacher 2008. Staudacher wählt für Reids Wahrnehmungstheorie die suggestive Bezeichnung „semiotischer Realismus".

22 Vgl. z.B. Essays 5.3, 395.

23 Dieser automatisierte Vorgang lässt sich jedoch durch viel Übung wieder bewusst machen und so von der eigentlich intentionalen Bezugnahme im Wahrnehmungsvorgang trennen. Reid führt das in Inquiry 6.3. am Beispiel eines Malers aus, der durch viel Übung diese unnatürliche Trennung erlernt hat. 
von einfachen Eigenschaften und Empfindungen sind die letzteren von großer Wichtigkeit: ${ }^{24}$

Es scheint mir, dass es natürliche Vorschläge [natural suggestions] gibt. Insbesondere, dass Empfindung uns den Begriff gegenwärtiger Existenz vorschlägt und die Überzeugung, dass das, was wir fühlen und wahrnehmen, gegenwärtig existiert; [...] Vermittels eines ähnlich natürlichen Prinzips schlägt uns der Anfang der Existenz oder irgendeines Wechsels in der Natur den Begriff der Ursache vor und bringt uns dazu, an seine Existenz zu glauben. Und in ähnlicher Weise [...] schlagen uns gewisse Tastempfindungen kraft der Konstitution unserer Natur die Ausdehnung [extension], Festigkeit und Bewegung vor, die in keiner Weise den Empfindungen ähnlich sind, obwohl sie bisher mit ihnen verwechselt wurden. (Inquiry 2.7, 111)

Empfindungen sind also nicht konventionelle, sondern natürliche Zeichen für Eigenschaften von Gegenständen. Dass Empfindungen für uns diese Rolle spielen können, ist teils angeboren, teils ein Produkt von Erfahrung und Gewohnheit. ${ }^{25}$ Einerseits kommt ihnen als natürlichen Zeichen überhaupt keine Intentionalität zu; sofern wir diese natürlichen Zeichen andererseits als Zeichen für etwas verwenden - eine Verwendungsweise, die zumindest zum Teil auch konventionell geprägt sein kann -, ist ihre daraus resultierende Intentionalität eine bloß abgeleitete, keine ursprüngliche Intentionalität. ${ }^{26}$

Empfindungen sind also nicht in demselben Sinne intentional wie Gedanken, Wahrnehmungen oder Erinnerungen, denen ursprüngliche Intentionalität zukommt. Der Zeichencharakter der Empfindungen gefährdet deshalb die Abgrenzung der Empfindungen von im eigentlichen Sinne intentionalen Zuständen nicht, sondern zwingt uns nur zur Klarstellung, dass Empfindungen weder als natürliche Zeichen, noch als Zeichen mit abgeleiteter Intentionalität ursprünglich intentionale Zustände sind.

Schließen wir nun unsere Diskussion der Abgrenzung von Empfindungen und intentionalen geistigen Zuständen, indem wir kurz die Frage aufgreifen, welche Eigenschaften die Empfindungen selbst haben und welche Eigenschaften wir als Eigenschaften der äußeren Gegenstände auffassen dürfen, die von den Empfindungen bezeichnet (signifyed) wer-

24 Den Begriff des Vorschlagens (suggestion) übernimmt Reid von Berkeley. Er führt ihn sogar ein, indem er dessen berühmtes Kutschenbeispiel zitiert. Vgl. Bd. 1, S. 352. Das Kutschenbeispiel ist für Reid Beispiel einer nicht-natürlichen, bloß erworbenen suggestion. (Vgl. ebd).

25 Vgl. Inquiry 6.4, 252.

26 Vgl. zu dieser Unterscheidung Searle 1983. 
den. Eine traditionelle Antwort auf diese Frage könnte auf die Unterscheidung primärer und sekundärer Qualitäten zurückgreifen: Sekundäre Qualitäten wahrgenommener Gegenstände, so ließe sich vielleicht behaupten, rufen Eigenschaften der Empfindungen hervor, die uns unmittelbar bewusst sind, primäre Qualitäten hingegen liefern uns ein unmittelbares Bewusstsein der Eigenschaften der wahrgenommenen Gegenstände ohne Rekurs auf Eigenschaften von Empfindungen.

Dass dies nicht Reids Auffassung sein kann, erhellt bereits das Zitat über die suggestion: Die dortigen Beispiele für Empfindungen, die natürliche Zeichen für Eigenschaften sind, sind allesamt klassische Beispiele primärer Qualitäten. An anderen Stellen macht Reid allerdings auch klar, dass auch sekundäre Qualitäten von Empfindungen vorgeschlagen werden. Gibt es dann für ihn überhaupt eine sinnvolle Unterscheidung primärer von sekundären Qualitäten oder lehnt er diese Unterscheidung mit Berkeley ab?

Reid hält die Unterscheidung zwischen primären und sekundären Qualitäten für eine substantielle Unterscheidung, die sich nicht etwa auf eine unfundierte Hypothese stützt. Sie hat ihren Ursprung vielmehr in der unmittelbaren Erfahrung bzw. der aufmerksamen Reflexion (attentive reflection) der Natur der Qualitäten und unseres Wissens von ihnen hat:

Sie werden dadurch unterschieden, dass wir von den primären Qualitäten vermittels unserer Sinne einen direkten und deutlichen Begriff [direct and distinct notion] haben; von den sekundären hingegen nur einen relationalen Begriff [relative notion], der dunkel bleiben muss, weil er bloß relational ist. Sie werden nur als unbekannte Ursachen oder Gelegenheiten gewisser Empfindungen vorgestellt, mit denen wir vertraut sind. (Essays 2.17, 314)

Von primären Qualitäten haben wir also einen direkten und deutlichen Begriff, d.h. unsere Sinne liefern uns Informationen darüber, was diese Qualitäten an sich selbst sind (Essays 2.17, 313). Von sekundären haben wir hingegen einen bloß relationalen Begriff, d.h. einen Begriff, der „strenggenommen überhaupt kein Begriff von einem Ding ist, sondern bloß von einer Relation, in der es zu etwas anderem steht“" (ebd. 314) - in diesem Fall uns selbst bzw. unseren Empfindungen. Da Empfindungen sekundärer Qualitäten nur in dieser Weise unbestimmt, wenn auch nicht unbestimmbar, ${ }^{27}$ über sich selbst hinausweisen, sind sie selbst ,der

27 Reid ist der Ansicht, dass die Naturwissenschaft dazu in der Lage ist, die Korrelate dieser Empfindungen zu erforschen (vgl. ebd. 185). Auf diese Weise, so kann man vermuten, könnten Empfindungen sekundärer Qualitäten auch erworbene Zeichen für Eigenschaften von Dingen werden. 
hauptsächliche Teil des Begriffs, den wir [von diesen Qualitäten] bilden“" (ebd. 315).

Wir stellen sie nur als das vor, was eine bestimmte Empfindung in uns hervorruft und können deshalb nicht über sie nachdenken, ohne an die Empfindung zu denken, die sie hervorrufen. (ebd.)

Anders die primären Qualitäten, auf die wir uns mit unserem direkten und deutlichen Begriff unmittelbar beziehen können. In diesem Fall gibt es, so Reid, keine Veranlassung dafür, uns bewusst auf die Empfindung zu beziehen, um uns vermittels dieser dann auf die Qualität zu beziehen, die sie hervorruft. Das heißt allerdings nicht, dass die fraglichen Empfindungen dann nicht mehr als Zeichen für die entsprechenden primären Qualitäten fungieren würden. Dieser Prozess ist nur vollständig automatisiert und daher weitgehend unbewusst: „Wenn eine primäre Qualität wahrgenommen wird, dann leitet die Empfindung unser Denken unmittelbar auf die bezeichnete (signifyed) Qualität und wird selbst vergessen.“ (ebd.)

Die Unterscheidung zwischen primären und sekundären Qualitäten wird bei Reid also nicht durch Ähnlichkeit oder Unähnlichkeit der Empfindung mit der Qualität, die sie hervorruft, definiert, wie etwa bei Locke; vielmehr sind sowohl sekundäre als auch primäre Qualitäten den Empfindungen völlig unähnlich, die sie hervorrufen. ${ }^{28}$ Allerdings geben uns die Empfindungen primärer Qualitäten einen unmittelbaren und deutlichen Begriff dieser Qualitäten, während die Empfindungen sekundärer Qualitäten gleichsam nur von sich selbst sprechen.

\subsubsection{Eine anti-repräsentationalistische Theorie der Wahrnehmung?}

Am Beginn dieser Überlegungen haben wir festgestellt, dass die Unterscheidung von Empfindung und Wahrnehmung in Reids Kritik der Ideentheorie einen ebenso wichtigen Platz einnimmt wie in seiner konstruktiven Wahrnehmungstheorie. Wenden wir uns deshalb nun zunächst dem zweiten Bestandteil dieser Unterscheidung und damit Reids Analyse der Wahrnehmung zu. Im abschließenden Teil dieser Überlegungen (vgl. unten 3.4) werden wir uns dann dem komplizierten Zusammenwirken von Empfinden und Wahrnehmen bei Reid widmen.

${ }^{28} \mathrm{Vgl}$. oben das Zitat bei der Einführung von suggestion. 
Reid fasst in einem berühmten Zitat aus den Essays drei wichtige Elemente der Wahrnehmung zusammen, nämlich Konzeption (conception) (alternativ auch Begriff [notion] oder Bild [image]), Unmittelbarkeit (immediacy) und Überzeugung (conviction oder belief):

Wenn wir uns nun also dem Akt unseres Geistes zuwenden, den wir als Wahrnehmung eines äußeren Sinnesgegenstandes [external object of sense] bezeichnen, so finden wir in diesem die folgenden drei Dinge: Erstens eine Vorstellung [conception] oder einen Begriff [notion] des wahrgenommenen Gegenstands; zweitens eine starke und unwiderstehliche Überzeugung [conviction] seiner Gegenwart; und drittens dass diese Überzeugung oder dieser Glaube [belief] unmittelbar sind und nicht das Ergebnis einer Überlegung [reasoning]. (Essays 2.5, 258)

Zurecht weist Van Cleve darauf hin, dass diese Aufzählung keine vollständige Definition von Wahrnehmung enthält. So fehlt beispielsweise der Verweis auf die Notwendigkeit einer Kausalkette zwischen dem wahrgenommenen Gegenstand und unserer Überzeugung. ${ }^{29}$ Und vor diesem Hintergrund muss an dieser Stelle auch offen bleiben, ob nicht der Zustand der Empfindung in dieser Kausalkette einen wesentlichen Beitrag zu leisten hat. Doch zunächst müssen wir klären, was die Elemente der dreifachen Charakterisierung jeweils zum Wahrnehmungsbegriff beitragen.

Beginnen wir mit dem Begriff der Überzeugung. Überzeugungen sind, genau wie Urteile, propositionale Entitäten: Sie weisen propositionale Struktur auf. ${ }^{30}$ Doch zusätzlich zu dieser propositionalen Struktur geht es Reid bei Überzeugung und Urteil auch noch um einen Vorgang des Zustimmens, der Bejahung, der Affirmation. Was in jedem Fall der Wahrnehmung affirmiert wird, ist die Existenz des wahrgenommenen Gegenstandes. Wir sind, so sagt er an anderer Stelle, ,unwiderstehlich von der Existenz [des wahrgenommenen Gegenstandes] überzeugt. Das ist immer der Fall, wenn wir uns sicher sind, dass wir ihn wahrnehmen." (Essays 2.5, 258) Wir mögen, so Reid weiter, bisweilen im Unklaren darüber sein, ob es sich bei einem geistigen Zustand tatsächlich um eine Wahrnehmung handelt; sofern wir aber sicher sind, dass es eine Wahrnehmung ist, sind wir auch sicher, dass der Gegenstand dieser Wahrnehmung existiert (ebd.).

Zwar beschränkt Reid in seinen Beispielen die fragliche Gewissheit auf die Gewissheit der Existenz des wahrgenommenen Gegenstandes, doch spricht nichts dagegen, diese Gewissheit auf dessen wahrgenom-

\footnotetext{
29 Van Cleve 2004, 106. Vgl. für die Bedeutung der Kausalkette z. B. Essays 2.2.

$30 \mathrm{Vgl}$. Essays 6.1, 414.
} 
mene Eigenschaften zu übertragen: Ich bin mir, um ein Beispiel Reids zu verwenden, nicht nur sicher, dass das Schiff, das ich sehe, existiert, sondern auch dass das, was ich da sehe, ein Schiff ist. ${ }^{31}$

Während Überzeugungen und Urteile wesentlich propositionale Entitäten sind, lässt sich ähnliches über die Vorstellung (conception) eines Gegenstands der Wahrnehmung oder den Begriff (notion) dieses Gegenstands nicht sagen. Die Vorstellung oder der Begriff wird in diesem Zusammenhang von Reid vielmehr der „einfachen Apprehension [simple apprehension]" (Essays 4.1, 360) angenähert. Was ist damit gemeint? ${ }^{32}$

Reid unterscheidet verschiedene Arten von Vorstellung. Jede Vorstellung ist ein Prozess: Dabei wird entweder sinnliches Material aus der Wahrnehmung oder der Erinnerung verwendet oder neue Begriffe werden aus alten zusammengesetzt. Im ersten Fall handelt es sich immer um die Vorstellung von Individuen, im zweiten Fall um die Vorstellung von Individuen oder von Universalien. Für die Analyse der Wahrnehmung ist zunächst die Vorstellung von Individuen interessant. ${ }^{33}$

Doch welche Art der individuellen Vorstellung hat Reid in der Aufzählung der Elemente der Wahrnehmung im Sinn? Die Vorstellung von Individuen vermittels des Arrangements innerer Bilder oder die Vorstellung von Individuen vermittels des Arrangements von Begriffen? Oder gibt es noch eine dritte Art der Vorstellung, die nur in einer mentalen Hinweisgeste besteht, wie Wolterstorff und Van Cleve meinen? ${ }^{34}$ Wie wir sehen werden, ist strenggenommen keine dieser Auffassungen von Reidschen Vorstellungen korrekt.

Die Konzeption vermittels des Arrangements von Begriffen können wir schnell ausschließen: Denn Reid verhandelt in diesem Zusammenhang ausschließlich das, was wir als ,knowledge by description“ bezeichnen würden. ${ }^{35}$ Und dieses Wissen setzt gerade keinen unmittelbaren Kontakt zum so beschriebenen Gegenstand voraus, wie er in der Wahrnehmung präsupponiert wird, die Reid ja grundsätzlich veridisch auffasst.

31 Reids Überzeugung entspricht also in etwa dem, was Peter Strawson einmal als Wahrnehmungsüberzeugung bezeichnet hat. Vgl. Strawson 1974a, 66.

32 Vgl. zum Folgenden Essays 4.1.

33 Auch die Vorstellung von Universalien ist für Wahrnehmung relevant, insbesondere für das, was Reid als mature state of conception bezeichnet. Vgl. Essays 6.3, 418.

34 Vgl. Wolterstorff 2001, Kap. 1 und Van Cleve 2003, 108.

35 Vgl. Van Cleve 2004, 108. 
Auch die Konzeption von Individuen vermittels des Arrangements innerer Bilder ist kaum in Reids Auffassung von Wahrnehmung integrierbar. Denn gerade die Zurückweisung einer Wahrnehmung innerer Bilder ist ein zentraler Bestandteil seiner Kritik an der Ideentheorie: Sie verletzt die Unmittelbarkeit der Wahrnehmung. Wenn wir deshalb in der Reidschen Wahrnehmung immer innere Bilder wahrnehmen würden, dann hätten wir gegenüber der Ideentheorie nichts gewonnen.

Wäre das Reids einziger Kritikpunkt, so ließe sich eine Bildchenauffassung von Vorstellung allerdings noch retten. Denn was zur Diskussion steht, ist ja nicht die Wahrnehmung innerer Bilder, sondern einfach das Haben innerer Bilder. Und in der Tat scheint Reid der Analogie zwischen Vorstellungen und Bildern in diesem Sinne etwas abgewinnen zu können:

Wenn ein Sprachunkundiger fragen sollte: Was heißt es, ein Ding vorzustellen [conceiving a thing]?‘, so wäre eine natürliche Antwort: Es heißt ein Bild von diesem Ding im Geist zu haben - und vermutlich könnten wir das Wort nicht besser erklären. Das zeigt, dass ,Vorstellung' und ,Bild eines Dinges im Geist' bedeutungsgleiche Ausdrücke sind. Das Bild im Geist ist demnach nicht der Gegenstand der Vorstellung, noch ist es eine Wirkung, die durch die Vorstellung als ihre Ursache hervorgerufen wurde. Es ist die Vorstellung selbst. Genau der geistige Zustand [mode of thinking], den wir als Vorstellung bezeichnen, kann ebenso wohl ein Bild im Geist genannt werden. (Essays 4.1, 363)

Das klingt so, als könnten wir die bilderzeugende Auffassung von Konzeption sehr wohl mit Reids Kritik an der Ideentheorie vereinbaren. Er vermeidet ja gerade den von ihm monierten Fehler, Bilder im Geist zum Gegenstand der Wahrnehmung zu machen und so die Unmittelbarkeit der Wahrnehmung zu gefährden: Bilder im Geist, Vorstellungen, wären vielmehr ein unverzichtbarer Bestandteil der Wahrnehmung, der den wahrgenommenen Gegenstand im Wortsinne abbildet und als Abbildung die Grundlage der Überzeugung von der Existenz des Wahrnehmungsgegenstands bildet.

Doch auch das kann nicht Reids Ansicht sein. Sehr deutlich schreibt er beispielsweise in seinen Essays:

Natürlich erleichtern es analoge Wörter und Ausdrücke, wie sie in allen Sprachen verwendet werden, um sich auf Vorstellungen zu beziehen, sie wörtlich zu nehmen. Wenn wir aber sorgfältig darauf achten, was uns in diesen Operationen des Geistes bewusst ist, so werden wir nicht mehr Anlass dafür finden, zu glauben, dass in unserem Geist wirklich Bilder existieren, als dass es dort [...] mechanische Maschinen gibt. (Essays 4.2, 373-374)

Keine Bilder im menschlichen Geist - deutlicher kann man, wie es scheint, kaum sein. Und dennoch finden wir bei Reid mehr von der 
Bilder-Auffassung von Vorstellungen, als Zitate wie dieses vermuten ließen.

Wie viel genau, das wird deutlich, wenn man sich genauer ansieht, wie Reid hinsichtlich der analogen Redeweise von Bildern im Geist die Disanalogie zwischen Bildern und Vorstellungen konstruiert. Zum Teil haben wir sie ja bereits ausgeführt: Vorstellungen funktionieren nicht wie Bilder, weil sie, erstens, keine Gegenstände der Wabrnehmung sind. Zweitens kann man bei Vorstellungen nicht zwischen dem Produkt und dem Vorgang der Produktion unterscheiden - Vorstellungen sind der Vorgang des Vorstellens, nicht dessen Produkt; Bilder hingegen sind nicht der Vorgang des Malens, sondern dessen Produkt. ${ }^{36}$ Drittens sind Vorstellungen ihrem Gegenstand nicht ähnlich, Bilder in der Regel schon. ${ }^{37}$

Reid expliziert andererseits die Analogie zwischen Vorstellungen und Bildern vor allem hinsichtlich der verschiedenen Arten von Bildern und der verschiedenen Arten von Vorstellungen: Bilder und Vorstellungen können reine Produkte der Einbildungskraft, also Phantasiegebilde, sein; sie können andere Vorstellungen kopieren oder replizieren; und sie können Vorstellungen oder Bilder, nach der Natur'sein, d.h. sie können natürliche Gegenstände, Individuen (individuals), vorstellen oder abbilden.

Im Kontext dieser Explikation der Analogie bezüglich der Arten von Vorstellungen und der Arten von Bildern bemerkt Reid ausdrücklich, dass darüber hinaus eine andere Analogie sehr stark ist, nämlich die Analogie zwischen dem Verfertigen von Bildern und Vorstellungen, die für Reid ja zunächst schlicht der Vorgang des Vorstellens sind: „Wir können eine starke Analogie zwischen dem Malen von Bildern und dem Vorstellen beobachten“" (Essays 4.1, 363). Worin genau besteht diese Analogie?

Anhand der von Reid hervorgehobenen Disanalogien können wir die Analogie zwischen Vorstellungen und dem Hervorbringen von Bildern zunächst folgendermaßen charakterisieren: Vorstellungen sind, erstens, der Vorgang des Vorstellens und nicht dessen Gegenstand, so wie das Malen des Bildes dessen Hervorbringung ist und nicht das Bild selbst. Vorstellungen sind, zweitens, der Vorgang des Vorstellens, so wie das Malen der Vorgang der Hervorbringung eines Bildes ist. Und Vorstellungen sind, drittens, dem Gegenstand der Vorstellung nicht ähnlich, so wie das

\footnotetext{
36 Vgl. Essays 4.1, 363.

37 Vgl. Essays 4.2, 374; Bd. 1, S. 423.
} 
Malen des Bildes dem Gegenstand des Bildes nicht ähnlich ist. Wie es scheint, besteht die Analogie zwischen Bildern und Vorstellungen für Reid demnach vor allem in einer Analogie zwischen dem Malen und dem Vorstellen.

Doch auch das kann nicht die ganze Wahrheit sein: Denn Reid bezeichnet nun einmal Vorstellungen auch als Bilder und er unterscheidet Arten von Vorstellungen gemäß den Arten von Bildern. Wenn es, wie Reid formuliert, keine Bilder gibt, „die vom Vorstellen des Gegenstandes unterschieden sind“ (Essays 4.2, 373; Hervorhebung J.H.) bzw. keine „Bilder im Geist, die vom Denken verschieden sind“ (ebd., 374; Hervorhebung J.H.), so heißt das eben auch, dass Vorstellungen in Analogie zu Bildern aufgefasst werden können. Bilder stellen ihren Gegenstand als etwas dar, indem sie ihn abbilden, ihm ähnlich sind, so Reids Auffassung. Reicht es für die Analogie zwischen Bildern und Vorstellungen vielleicht aus, dass Vorstellungen etwas darstellen oder sich auf etwas beziehen, obwohl sie ihm unähnlich sind?

Das wäre eine Auffassung von Vorstellung, die - so wie das Malen von Bildern - der Prozess des Hervorbringens eines geistigen Bildes ist, und gleichzeitig ein Prozess, der selbst bereits ein Bild ist, indem er sich auf etwas als etwas bezieht. Dabei ist die Vorstellung, oder besser: der Vorstellungsprozess, selbst dem Gegenstand unähnlich, auf den wir uns in der Wahrnehmung beziehen, deren wesentlicher Teil sie ist.

Diese Sichtweise erfasst sicherlich einen wesentlichen Teil von Reids Bildanalogie; allerdings greift auch sie noch zu kurz. Um das zu sehen, müssen wir die Empfindungen wieder in den Blick nehmen und ihr Verhältnis zur Vorstellung. Empfindungen sind zunächst Zeichen für einzelne Eigenschaften; Vorstellungen sind hingegen Vorstellungen von Gegenständen, d.h. Entitäten, denen eine Vielzahl von Eigenschaften zukommt. Reid schuldet uns deshalb eine Theorie darüber, wie wir von den Empfindungen von einfachen Qualitäten zur Vorstellung - und damit mittelbar zur Wahrnehmung - von Komplexen von Qualitäten übergehen können.

Reid hat eine solche Theorie nicht ausbuchstabiert; dennoch kann uns die Bildanalogie hier weiterhelfen. Wir haben weiter oben die Interpretation abgelehnt, die Reids Auffassung von Vorstellung als bilderzeugendes Verfahren im Sinne einer Abbildung des wahrgenommenen Gegenstandes im Geiste beschreibt. Nun können wir diese Konzeption aber so modifizieren, dass daraus eine Auffassung wird, die mit Reids Ablehnung der Bildchenauffassung zumindest verträglich erscheint und eine Antwort auf die Frage der Gegenstandskonstitution im Reidschen 
Geiste zulässt: Reid könnte Vorstellungen als Verfahren der Erzeugung von komplexen geistigen Zuständen auffassen, die den wahrgenommenen Gegenständen qua Zustände natürlich nicht ähnlich sein können, aber qua Arrangementvorgänge von Zeichen für Eigenschaften von Dingen - d.h. als Arrangementvorgänge von Empfindungen -, kausal und semiotisch mit ihnen in systematischer Verbindung stehen.

Vorstellungen von Gegenständen wären demnach die Konstruktionsprozesse von Eigenschaftskomplexen aus primären Qualitäten und (Empfindungen von) sekundären Qualitäten. ${ }^{38}$ Diese Eigenschaftskomplexe funktionieren nicht wie Bilder hinsichtlich derjenigen Eigenschaften von Gegenständen der Wahrnehmung, die diese Eigenschaften selbst so haben, wie wir sie wahrnehmen (primäre Qualitäten); und sie wären wie Bilder hinsichtlich der Eigenschaften, die die wahrgenommenen Gegenstände selbst nicht haben (Empfindungen sekundärer Qualitäten). Als genau dieser Eigenschaftskomplex würde sich ihre Existenz auf das Haben der Vorstellung, den Vorstellungsprozess, beschränken, da nur in ihm beide Eigenschaftsarten in der beschriebenen Weise zusammenwirken können. ${ }^{39}$

Ein für unsere Zwecke wichtiger Einwand lässt sich gegen diese Rekonstruktion vorbringen: Wird durch die Einbeziehung dieses Zwischenglieds nicht die Unmittelbarkeitsforderung verletzt, d.h. der dritten Bestandteil von Reids Wahrnehmungscharakterisierung? Ein wesentlicher Teil der Antwort wurde bereits mit Reids am alltäglichen Sprachgebrauch orientierten Common-Sense-Analyse des Begriffs der Vorstellung geleistet: Denn Reid insistiert in dieser Analyse ja darauf, dass die Vorstellung nicht ein vom Bild im Geiste verschiedener Akt der Aufmerksamkeit ist. Ein solcher Akt der Aufmerksamkeit wäre, sofern er für die Wahrnehmung eines äußeren Gegenstandes notwendig wäre, tatsächlich ein Zwischenglied im Wahrnehmungsprozess, das die Unmittelbarkeit der Wahrnehmung des äußeren Gegenstandes gefährden würde: Wir stünden in einer epistemischen oder kognitiven Relation zu dem Bild des Gegenstandes, das so gleichsam zum epistemischen Mittler zwischen dem Gegenstand und unserer Wahrnehmung würde.

Doch dieser Verdacht ist unbegründet: Von einer derartigen epistemischen Mittleraufgabe kann in der skizzierten Auffassung keine Rede

38 Vgl. zu Reids Auffassung der Primär/Sekundär-Unterscheidung oben S. 452-453.

39 Diese Rekonstruktion passt übrigens gut zu Staudachers Differenzierung verschiedener Dimensionen des Reidschen Realismus hinsichtlich der Unterscheidung primärer und sekundärer Qualitäten. Vgl. Staudacher 2008, 340-344. 
sein. Denn dieses innere Bild ist, sofern die richtige Form der kausalen Abhängigkeit von dem wahrgenommenen Gegenstand besteht ${ }^{40}$, nichts anderes als das Vorstellen des Gegenstands selbst, das - nach offizieller Reidscher Doktrin: zusammen mit der Überzeugung - nichts anderes ist als die unmittelbare Wabrnehmung des Gegenstands.

\subsubsection{Die Rolle der Empfindung in der Wahrnehmung}

Wenn Vorstellungen tatsächlich in der beschriebenen Weise charakterisiert werden können, d.h. als unmittelbar auf den wahrgenommenen Gegenstand bezogene innere Bilder, die sinnliche und begriffliche Aspekte in sich vereinen, stellt sich natürlich die Frage, ob nicht in diese Aspekte der Wahrnehmung ein Aspekt der Empfindung wesentlich eingeht. Was sonst, so ist man versucht zu fragen, könnte in Reids Wahrnehmungstheorie das sinnliche Material für die Vorstellung liefern?

In der Tat sieht es an vielen Stellen, sowohl im früheren Inquiry als auch in den Essays, so aus, als wäre Reids Bild des Wahrnehmungsvorgangs etwa folgendes:

affiziert

affiziert

Wahrnehmungs-
gegenstand $\quad \rightarrow \quad$ Empfindung $\rightarrow \quad \begin{aligned} & \text { Vorstellung \& } \\ & \text { Überzeugung }\end{aligned}$

Dieses Bild ist nicht unplausibel: Es wird den bisherigen Überlegungen gerecht und fügt die Empfindungen an der Stelle in das Bild ein, an der das gewonnene Bild der Vorstellung uns dies erwarten lässt.

Allerdings ist das Bild auch nicht unproblematisch und wirft einige wichtige Fragen im Zusammenhang von Reids Theorie der Wahrnehmung auf. Die zentrale Frage betrifft dabei die Notwendigkeit der Empfindung: Gibt es nicht Fälle von Wahrnehmung, oder sind solche nicht zumindest vorstellbar, in denen Empfindung keine Rolle spielt? Die positive Beantwortung dieser Frage würde die Bemühung um Empfindung als Teil der Wahrnehmung von vorneherein zum Scheitern verurteilen.

Wenigstens zwei Aspekte von Reids Theorie scheinen hier Schwierigkeiten zu bereiten: Erstens scheint er es für vorstellbar zu halten, dass Wahrnehmung auch ohne Empfindung möglich wäre; und zweitens

40 Vgl. oben S. 450. 
scheint er, stärker noch, sogar tatsächliche Fälle zu beschreiben, in denen die Empfindung ausdrücklich keine Rolle spielt: nämlich in der visuellen Wahrnehmung von Formen (visible form).

Den ersten Fall können wir schnell beiseite lassen: Bloße Vorstellbarkeit sollte in diesem Zusammenhang keine Rolle spielen, da andernfalls auch die Rolle der Vorstellung (weitgehend unabhängig von der gewählten Auffassung!) verzichtbar erscheint. Reid scheint es im Zusammenhang der Analyse unserer Wahrnehmung allgemein um Deskription zu gehen. Begriffsanalyse, die die erforderlichen Notwendigkeiten implizieren würde, ist hier immer nur eine Form des deskriptiven Zugangs. Eine andere, gleichfalls legitime und von Reid häufig hervorgehobene Form des deskriptiven Zugangs ist die Methode sorgfältiger Introspektion. Und diese scheint seiner Ansicht nach durchaus der Empfindung den Platz zuzuschreiben, den sie in der obigen Grafik innehat.

Wie sieht es mit den Fällen aus, in denen Wahrnehmung ohne Empfindung aufzutreten scheint? Dieses Problem wiegt tatsächlich schwer. Visible forms dienen Reid als physische Zeichen für externe Körper, die sie unter verschiedenen Perspektiven vermittels der eigenen Ausdehnungseigenschaften als ausgedehnte Körper abbilden. Diese Perspektivität kann deshalb auch keine Empfindungseigenschaft sein, da Empfindungen selbst nicht ausgedehnt sind. Sind visible forms also ein Beispiel für Wahrnehmung ohne Empfindung?

Eine Reaktionsmöglichkeit bestünde darin, darauf zu verweisen, dass diese Beobachtungen sich ausschließlich im früheren Inquiry finden und in den Essays nicht wiederholt werden. Allerdings ist die textliche Situation nicht ganz eindeutig: Reid spricht auch in den Essays ausführlich über visible figure - ohne dabei jedoch die Behauptung bezüglich Empfindung zu wiederholen. ${ }^{41}$ Das Verhältnis bleibt also unklar und lässt deshalb interpretatorischen Spielraum.

In exegetischer Hinsicht spricht gegen diese Interpretation der Wahrnehmung visueller Formen als empfindungslose Wahrnehmung allerdings, dass Reid den Ausdehnungseigenschafen, wie wir gesehen haben, explizit Empfindungen zuordnet. ${ }^{42}$ Und Ausdehnungseigenschaften schließen sicherlich die Formeigenschaften ein. Vor diesem Hintergrund

\footnotetext{
41 Vgl. Essays 2.19, $324 \mathrm{ff}$.

42 Vgl. oben S. 453.
} 
scheint es unplausibel, Reid auch noch in den Essays die Form-Auffassung aus dem Inquiry zu unterstellen. ${ }^{43}$

Darüber hinaus lässt sich aber auch in der folgenden Weise systematisch argumentieren: Visuelle Formwahrnehmung im von Reid intendierten Sinne ist uns niemals allein gegeben. Wir nehmen nie bloßvisuelle Form wahr, sondern immer visuelle Form mit bestimmten Farbeigenschaften etc. Und es verhält sich keineswegs so, dass wir etwa bloß faktisch keine derartigen Fälle bloßer visueller Formwahrnehmung hätten: vielmehr ist visuelle Formwahrnehmung immer abhängig von Kontrastwahrnehmung, da wir ohne die Wahrnehmung von Kontrasten keine Form wahrnehmen würden. ${ }^{44}$ Die Wahrnehmung von Kontrasten ist aber eine durch Empfindungen vermittelte. Selbst wenn also die visuelle Gestalt keine Eigenschaften enthielte, die Empfindungen hervorrufen, so wäre doch die Wahrnehmung der visuellen Gestalt ohne begleitende Empfindungen unmöglich.

Abschließend können wir deshalb über die Rolle der Empfindungen in Reids Konzeption der Wahrnehmung sagen: Gerade weil Empfindungen so verschieden von intentionalen geistigen Zuständen sind, sind sie ein wesentlicher Bestandteil seiner realistischen Wahrnehmungstheorie. Empfindungen sind wesentliche Bestandteile der Vorstellungen und damit wesentliche Bestandteile der Wahrnehmung.

43 Das Argument mit dem Blinden, der einen Begriff der visuellen Form entwickeln könnte, wenn er über die nötigen mathematisch-naturwissenschaftlichen Kenntnisse verfügt, das Reid selbst im Inquiry (...) anführt, wird zwar immer wieder als Grund dafür angeführt, das Reid diese These mit guten Gründen dauerhaft vertreten hat, ist abergemäß seiner eigenen Überlegungen in den Essays - offensichtlich unbefriedigend: Denn dort schildert Reid ein naturwissenschaftliches Verständnis auch des Zusammenhangs von sekundären Qualitäten und ihren Empfindungen - ohne doch einzuräumen, dass wir nun ein Verständnis von den Empfindungen sekundärer Qualitäten hätten, das dem entspräche, das wir haben, wenn wir selbst diese Empfindungen empfunden haben. Analog sollte auch der Blinde die relevanten Qualia entbehren.

44 Ähnlich auch Staudacher 2007, 336-337. 